\title{
Vaspin protects against LPS-induced ARDS by inhibiting inflammation, apoptosis and reactive oxygen species generation in pulmonary endothelial cells via the Akt/GSK-3ß pathway
}

\author{
DI QI $^{1 *}$, DAOXIN WANG ${ }^{1 *}, \mathrm{CHUNRONG} \mathrm{ZHANG}^{2}, \mathrm{XUMAO} \mathrm{TANG}^{1}, \mathrm{JING} \mathrm{HE}^{1}$, \\ YAN ZHAO $^{1}$, WANG DENG ${ }^{1}$ and XINYU DENG ${ }^{1}$ \\ ${ }^{1}$ Department of Respiratory Medicine, Second Affiliated Hospital of Chongqing Medical University, \\ Chongqing 400010; ${ }^{2}$ Department of Emergency, Yongchuan Affiliated Hospital of \\ Chongqing Medical University, Chongqing 402160, P.R. China
}

Received December 21, 2016; Accepted September 27, 2017

DOI: $10.3892 / \mathrm{ijmm} .2017 .3176$

\begin{abstract}
Acute respiratory distress syndrome (ARDS) is characterized by uncontrolled extravasation of protein-rich fluids, which is caused by disruption and dysfunction of the barrier of pulmonary endothelial cells (ECs). Visceral adipose tissue-derived serine protease inhibitor (vaspin) is a novel adipokine with pleiotropic properties, which has been reported to exert beneficial effects against obesity-associated systemic vascular diseases; however, its effects on ARDS remain unknown. In the present study, mice were subjected to systemic administration of adenoviral vector expressing vaspin (Ad-vaspin) to examine its effects on lipopolysaccharide (LPS)-induced ARDS in vivo. Histological analysis was then conducted, and cytokine [tumor necrosis factor (TNF)- $\alpha$, interleukin (IL)-6 and IL-10] levels, and intercellular cell adhesion molecule-1 (ICAM-1) and adherens junctions (AJs)
\end{abstract}

Correspondence to: Professor Chunrong Zhang, Department of Emergency, Yongchuan Affiliated Hospital of Chongqing Medical University, 439 Xuanhuan Road, Chongqing 402160, P.R. China E-mail: cqmuzhangchunrong@163.com

Abbreviations: ARDS, acute respiratory distress syndrome; CAD, coronary artery disease; IJV, internal jugular vein; LPS, lipopolysaccharide; Ad-vaspin, adenoviral vector expressing vaspin; Ad- $\beta$-gal, adenoviral vector expressing $\beta$-galactosidase; rh-vaspin, recombinant human vaspin; ECs, endothelial cells; EBDA, Evans blue dyed-albumin; W/D, wet/dry; HPMECs, human pulmonary microvascular endothelial cells; SMCs, smooth muscle cells; CCK-8, Cell Counting kit-8; TUNEL, TdT-mediated dUTP nick end labeling; FCM, flow cytometry; FITC, fluorescein isothiocyanate; PI, propidium iodide; ICAM, intercellular cell adhesion molecule; VCAM, vascular cell adhesion molecule; MCP-1, monocyte chemotactic protein-1; AJ, adherens junction; DHE, dihydroethidium; ROS, reactive oxygen species; NADPH, nicotinamide adenine dinucleotide phosphate

Key words: acute respiratory distress syndrome, vaspin, endothelial cells, inflammation, apoptosis, reactive oxygen species, Akt/GSK-3 $\beta$ expression were detected. In addition, human pulmonary microvascular ECs (HPMECs) were treated with recombinant human (rh)-vaspin to further investigate its molecular basis and underlying mechanism. The mRNA expression levels of inflammatory cytokines (TNF- $\alpha$ and IL-6) and endothelial-specific adhesion markers [vascular cell adhesion molecule-1 and E-selectin], activation of nuclear factor- $\kappa \mathrm{B}$, and cell viability and apoptosis were then examined. Furthermore, the expression of AJs and organization of the cytoskeleton, as well as expression and activity of nicotinamide adenine dinucleotide phosphate (NADPH) oxidase and generation of reactive oxygen species (ROS) were determined. The results indicated that Ad-vaspin protected against LPS-induced ARDS by alleviating the pulmonary inflammatory response and pulmonary EC barrier dysfunction in mice, which was accompanied by activation of the protein kinase $\mathrm{B}$ (Akt)/glycogen synthase kinase (GSK)-3 $\beta$ pathway. In addition, pretreatment of HPMECs with rh-vaspin attenuated inflammation, apoptosis and ROS generation without alterations in AJs and cytoskeletal organization following LPS insult, which was accompanied by activation of the Akt/GSK3 $\beta$ pathway. In conclusion, the present study demonstrated that vaspin protects against LPS-induced ARDS by reversing EC barrier dysfunction via the suppression of inflammation, apoptosis and ROS production in pulmonary ECs, at least partially via activation of the Akt/GSK $3 \beta$ pathway. These findings provide evidence of a causal link between vaspin and EC dysfunction in ARDS, and suggest a potential therapeutic intervention for patients with ARDS.

\section{Introduction}

Acute respiratory distress syndrome (ARDS) is a severe medical condition, which is associated with a high mortality rate, and is characterized by noncardiogenic pulmonary edema and hypoxemia $(1,2)$. Although ARDS is not a classified pulmonary vascular disease, disruptions in endothelial barrier integrity and dysfunction of endothelial barrier permeability are considered the pathological hallmarks of ARDS, particularly at the very early stage of ARDS development $(3,4)$. 
It is well known that the development of ARDS is associated with various predisposing risk factors, among which obesity has been identified in recent epidemiological studies (5-8). Obesity, particularly visceral obesity, has been reported to impair systemic vasculature and is involved in the initiation and progression of cardiovascular diseases $(9,10)$. Adipose tissue is considered a significant endocrine organ that is capable of crosstalk with peripheral organs via diverse bioactive molecules known as adipokines, which participate in the progression of diverse vascular disorders (11-13). Although different from the well-documented effects of obesity on cardiovascular disease, the relationship between obesity and ARDS has proven to be considerably complex; clinical and experimental data have focused on pertinent physiological alterations in obesity and have highlighted the pivotal role of pulmonary vascular 'priming' and neutrophil functional impairment at baseline $(8,14)$. Since persistent, low-grade inflammation as a result of fat accumulation impairs systemic blood vessels and contributes to the development of various obesity-associated vascular diseases, emerging evidence has suggested that obesity alters ARDS pathogenesis by 'priming' the pulmonary endothelial barrier for insult and injury, as well as amplifying the early inflammatory response, thus lowering the threshold required to initiate ARDS via dysregulated adipokine production in obesity. Previous studies have demonstrated that circulating adipokine levels are associated with the initiation and progression of ARDS (15-18).

Visceral adipose tissue-derived serine proteinase inhibitor (vaspin) is identified as a favorable adipokine that is secreted from visceral white adipose tissues, which is associated with glucose tolerance and chronic inflammation $(19,20)$. Vaspin has been reported to counteract the pathogenesis of various obesity-related vascular complications via its anti-inflammatory and anti-apoptotic properties. In a human study, serum vaspin levels were demonstrated to be lower in patients with coronary artery disease (CAD) compared with in the control individuals; this tendency was confirmed in control individuals with higher systolic blood pressure compared with in control individuals with normal blood pressure, thus indicating that vaspin may be a predictor of CAD (21). Furthermore, a previous study reported that lower fasting vaspin levels were correlated with ischemic vascular events in the last 3 months in patients with carotid stenosis compared to those with asymptomatic stenosis (22). Decreased serum vaspin levels were also observed in patients with ankylosing spondylitis and were associated with flow-mediated dilation levels, thus indicating that vaspin may be a marker for detecting early stage atherosclerosis in patients with ankylosing spondylitis (23). Vaspin is believed to serve a local and endocrine role in the initiation and development of vascular disorders by affecting endothelial cells (ECs), thus disrupting vascular homeostasis. A previous study suggested that vaspin, as a ligand for the cell surface $78 \mathrm{kDa}$ glucose-regulated protein (GRP78)/voltage-dependent anion channel complex in ECs, exerted beneficial effects on diabetic vascular complications by promoting proliferation and inhibiting apoptosis under the diabetic milieu via a protein kinase B (Akt)-dependent mechanism (24). It has also been demonstrated that vaspin may significantly attenuate methylglyoxal-induced cell death and reactive oxygen species (ROS) generation in human umbilical vein ECs (HUVECs) (25). Treatment with vaspin also significantly decreased tumor necrosis factor (TNF)- $\alpha$-induced activation of nuclear factor $(\mathrm{NF})-\kappa \mathrm{B}$, as well as the expression of the adhesion molecules intercellular cell adhesion molecule-1 (ICAM-1), vascular cell adhesion molecule-1 (VCAM-1), E-selectin and monocyte chemotactic protein-1 (MCP-1) in human aortic ECs (26). Furthermore, vaspin is capable of preventing apoptosis induced by free fatty acids through upregulation of the phosphoinositide 3-kinase (PI3K)/Akt signaling pathway in vascular ECs, suggesting the beneficial effects of vaspin on obesity-associated vascular diseases (27). A recent study also indicated that vaspin was able to inhibit the progression of atherosclerotic plaques in apolipoprotein $\mathrm{E}^{-/-}$mice by suppressing endoplasmic reticulum stress-induced macrophage apoptosis (28).

Collectively, these data suggested that vaspin, as a pleiotropic adipokine capable of exerting anti-inflammatory, anti-apoptotic and antioxidant effects on ECs, may exert a favorable role against the progression of obesity-associated vascular complications. Although the pathogenesis of ARDS is considerably complex, hyperpermeability of the pulmonary EC barrier is a pathological hallmark of ARDS at the very early stage, in which obesity, inflammation, apoptosis and ROS serve important roles. Therefore, it may be hypothesized that vaspin contributes to the protection of ARDS via its endothelial-protective effects. Nevertheless, to the best of our knowledge, no previous studies have assessed the effects of vaspin on pulmonary ECs, specifically in the setting of ARDS.

Therefore, the present study aimed to investigate the effects of vaspin on lipopolysaccharide (LPS)-induced ARDS in vivo and in vitro, and to further explore the molecular basis and potential mechanisms underlying these effects. This study aimed to provide a novel insight into the crosstalk between vaspin and ARDS, particularly focusing on the pulmonary EC barrier.

\section{Materials and methods}

Chemicals and reagents. LPS (Escherichia coli LPS serotype 0111:B4), sodium pentobarbital, Evans blue dye and dihydroethidium (DHE), goat serum (G9023) and bovine serum albumin (A2153) were purchased from Sigma-Aldrich (Merck KGaA, Darmstadt, Germany). Recombinant human vaspin (rh-vaspin) protein was purchased from GeneTex, Inc. (Irvine, CA, USA). The following primary antibodies were purchased from Cell Signaling Technology, Inc. (Danvers, MA, USA): Anti-ICAM-1 (cat. no. 4915), anti-NF- $\kappa$ B Rel (cat. no. 8242), anti-phosphorylated (p)-NF-кB Rel (Ser536; E1Z1T; cat. no. 13346), anti-Akt (cat. no. 4685) and anti-p-Akt (Ser473; cat. no. 4060), anti-rabbit IgG, HRP-linked antibody (cat. no. 7074) and Alexa Fluor 488-labeled anti-rabbit secondary antibodies (cat.no.4412).Anti-vascularendothelial(VE)-cadherin (ab205336) and anti-NADPH oxidase antibodies (ab133303) were purchased from Abcam (Cambridge, UK). Anti-GAPDH (cat. no. AP0063), anti- $\beta$-catenin (cat. no. BS6879), anti-glycogen synthase kinase (GSK)-3 $\beta$ (cat. no. BS1402) and anti-p-GSK3 $\beta$ (Ser9; cat. no. BS4084P) antibodies were purchased from Bioworld Technology (Nanjing, China). Adenoviral vectors (Ad) expressing $\beta$-galactosidase (Ad- $\beta$-gal) and full-length vaspin (Ad-vaspin) were constructed by Shanghai GeneChem Co., Ltd. (Shanghai, China) (primer sequences available upon request). Ad- $\beta$-gal was used as a control. 
LPS-induced ARDS model in mice. C57BL/6 mice (weighing 20-25 g; 10 weeks old; male; of specific-pathogen-free grade; Department of Laboratory Animal Center, Chongqing Medical University, Chongqing, China) were housed under light-controlled conditions (12-h light/dark cycle) at room temperature $\left(25^{\circ} \mathrm{C}\right)$ with $60 \%$ humidity and were granted ad libitum access to food and water. All animal experimental protocols were implemented in accordance with the instructions of the National Institutes of Health Guide for the Care and Use of Laboratory Animals (29). The present study was approved by the Ethics Committee of the Second Affiliated Hospital of Chongqing Medical University (Chongqing, China). Mice were randomly assigned to 4 groups as follows: the control group not pre-treated with Ad-vaspin ( $\mathrm{n}=24)$, the control group pre-treated with Ad-vaspin $(n=24)$, the ARDS group not pre-treated with Ad-vaspin and the ARDS group treated with Ad-vaspin $(n=24)$. Mice were anesthetized with sodium pentobarbital $(50 \mathrm{mg} / \mathrm{kg}$, i.p.) prior to exposure of the trachea and right internal jugular vein (IJV). Subsequently, $3 \times 10^{7}$ PFU Ad-vaspin or Ad- $\beta$-gal per mouse was injected into the IJV for 3 days prior to LPS or vehicle (PBS) intratracheal instillation. Mice were anesthetized with sodium pentobarbital $(50 \mathrm{mg} / \mathrm{kg}$, i.p.). As described previously (18), to establish a mouse model of ARDS, mice were intratracheally instilled with $5 \mathrm{mg} / \mathrm{kg}$ LPS (E. coli LPS serotype 0111:B4) in $50 \mu 1$ sterile PBS, or PBS alone (control group) using an 18-G catheter. The mice were sacrificed $4 \mathrm{~h}$ after the LPS injection and lung tissues were harvested and stored at $-80^{\circ} \mathrm{C}$ until further analysis.

Lung histological evaluation. Left lung lobes were harvested, fixed in $3.7 \%$ paraformaldehyde ( $24 \mathrm{~h}$ at room temperature), embedded in paraffin wax and cut into 5- $\mu \mathrm{m}$ sections. Subsequently, the sections were stained with hematoxylin and eosin. Histological lung injury in each mouse was evaluated in 5 random fields (x200 and x400 magnification) using an inverted microscope (TE2000-U; Nikon Corporation, Tokyo, Japan). A standardized scoring system, published by the American Thoracic Society (30), was used to assess histological lung injury in mice.

ELISA. Lung homogenates were used to determine the levels of TNF- $\alpha$ (MTA00B), interleukin (IL)-6 (M6000B) and IL-10 (M1000B) using commercially available ELISA kits (R\&D Systems, Inc., Minneapolis, MN, USA) according to the manufacturer's protocols. In cultured cells, basal levels of secretion were determined by measuring TNF- $\alpha$, IL- 6 and IL-10 concentration in cell lysates. The effectiveness of the adenoviral vector expression system was confirmed by measuring mean plasma vaspin concentrations in the treated mice using a commercial ELISA kit (DSA120; R\&D Systems, Inc.).

Immunohistochemistry. Mouse left lung tissue sections from each group were deparaffinized with xylene, rehydrated in gradient ethanol and incubated in $3 \% \mathrm{H}_{2} \mathrm{O}_{2}$ at $37^{\circ} \mathrm{C}$ for $15 \mathrm{~min}$. Subsequently, the sections were rinsed three times in PBS (10 min/wash). Antigen retrieval was performed by immersing the slices in citrate buffer in a microwave at $96^{\circ} \mathrm{C}$ for $30 \mathrm{~min}$. Tissues were blocked with goat serum albumin in an incubator at room temperature for $1 \mathrm{~h}$ and the sections were then incubated with anti-ICAM-1 primary antibodies
(1:50 dilution) at $4^{\circ} \mathrm{C}$ overnight. Sections were washed a further three times with PBS (10 min/wash) and were incubated with a biotin-labeled secondary antibody (1:1,000; ZB-2010; ZSBIO Biotech Co., Ltd., Beijing, China) at $37^{\circ} \mathrm{C}$ for $30 \mathrm{~min}$, and were then stained with DAB. Sections were counterstained with hematoxylin, dehydrated in gradient ethanol, vitrified with xylene and sealed with neutral resins. Images were captured using an inverted microscope (TE2000-U; Nikon Corporation).

Measurement of Evans blue-dyed albumin (EBDA) concentrations in lung tissue. Pulmonary capillary permeability was assessed by determining EBDA concentrations. The right IJV of mice was injected with EBDA $(30 \mathrm{mg} / \mathrm{kg})$. Then mice were sacrificed and lungs free of blood were excised, weighed and homogenized in $1 \mathrm{ml}$ PBS, after which they were extracted in $2 \mathrm{ml}$ formamide $\left(24 \mathrm{~h}, 60^{\circ} \mathrm{C}\right)$ and centrifuged at 5,000 $\mathrm{x} \mathrm{g}$ for $30 \mathrm{~min}$ at $20^{\circ} \mathrm{C}$. The absorbance of the supernatants was measured by spectrophotometry at 620 and $740 \mathrm{~nm}$, plotted against a standard curve, normalized, and converted to $\mu \mathrm{g}$ EBDA/g lung tissue.

Analysis of BALF. A trimmed $18-\mathrm{G}$ catheter was inserted into the trachea. A syringe was connected to the catheter, and $1 \mathrm{ml}$ of sterile normal saline was infused into the airway. Four hours after LPS administration, BALF was collected by the intratracheal instillation of $1 \mathrm{ml}$ of sterile normal saline followed by repeated aspiration 3 times and centrifugation at $500 \mathrm{xg}$ for $10 \mathrm{~min}$ at $4^{\circ} \mathrm{C}$. According to manufacturer's instructions, the protein concentrations in the BALF supernatants were determined using a bicinchoninic acid protein assay (BCA) kit (KeyGen Biotech Co., Ltd., Nanjing, China).

Wet/dry (W/D) lung weight ratio. The right upper lung lobes were harvested and weighed to determine wet lung weight. Subsequently, tissues were dried in an oven at $80^{\circ} \mathrm{C}$ for $24 \mathrm{~h}$ and were weighed again to calculate the W/D ratios.

EC culture. Human pulmonary microvascular ECs (HPMECs) were cultured in EC medium (both ScienCell Research Laboratories, Inc., San Diego, CA, USA) supplemented with $10 \%$ fetal bovine serum (FBS; cat. no. 0025), 1\% endothelial cell growth supplement (ECGS; cat. no. 1052) and 1\% penicillin/streptomycin (P/S; cat. no. 0503) (all from ScienCell Research Laboratories, Inc.) in a $5 \% \mathrm{CO}_{2}$ incubator at $37^{\circ} \mathrm{C}$. Cells between passages 4 and 10 were grown as a monolayer and starved (1\% serum) for $6 \mathrm{~h}$ prior to each treatment. Human serum concentrations of vaspin are reported to range between 0.1 and $100 \mathrm{ng} / \mathrm{ml}$; therefore, $10 \mathrm{ng} / \mathrm{ml}$ vaspin was used in the present study. Cells were pretreated with rh-vaspin $(10 \mathrm{ng} / \mathrm{ml})$ or PBS as a control for $24 \mathrm{~h}$, after which they were washed with PBS and exposed to LPS or vehicle (PBS) at $100 \mathrm{ng} / \mathrm{ml}$ for $2 \mathrm{~h}$. Cell lysates were collected for subsequent analysis at the indicated time intervals.

EC monolayer permeability assay. Permeability was determined based on the paracellular permeability of $70 \mathrm{kDa}$ fluorescein isothiocyanate (FITC)-dextran into the lower chamber as described previously (18). Briefly, HPMECs were grown on $0.4 \mu \mathrm{m}$ Transwell inserts at $1 \times 10^{5}$ cells per well. Following the indicated time interval for each treatment, $0.5 \mathrm{ml}$ FITC-dextran $(1 \mathrm{mg} / \mathrm{ml})$ was added to the upper wells, and $1.5 \mathrm{ml}$ medium was 
Table I. Polymerase chain reaction primer sequences.

\begin{tabular}{lcc} 
Gene & Forward primer $\left(5^{\prime} \rightarrow 3^{\prime}\right)$ & Reverse primer $\left(5^{\prime} \rightarrow 3^{\prime}\right)$ \\
\hline GADPH & AGGTCGGTGTGAACGGATTTG & TGTAGACCATGTAGTTGAGGTCA \\
TNF- $\alpha$ & CCCTCACACTCAGATCATCTTCT & GCTACGACGTGGGCTACAG \\
IL-6 & TAGTCCTTCCTACCCCAATTCC & TTGGTCCTTAGCCACTCCTTC \\
VE-cadherin & CACTGCTTTGGGAGCCTTC & GGGGCAGCGATTCATTTTCT \\
$\beta-$-atenin & CACAAGCAGAGTGCTGAAGGTG & GATTCCTGAGAGTCCAAAGACAG
\end{tabular}

IL-6, interleukin-6; TNF- $\alpha$, tumor necrosis factor- $\alpha$; VE-cadherin, vascular endothelial-cadherin.

added to the bottom chamber. Following $1 \mathrm{~h}$ incubation in the dark, $50 \mu \mathrm{l}$ medium was aspirated and absorbance was measured using a luminometer (BioTek Instruments, Inc., Winooski, VT, USA) at an excitation wavelength of $488 \mathrm{~nm}$ and an emission wavelength of $520 \mathrm{~nm}$. The basal FITC-dextran permeability for unstimulated monolayers was set at $100 \%$.

EC viability assay. Cell viability was measured using Cell Counting kit-8 (CCK-8). Cell suspensions of each group were seeded in 96-well plates at $2 \times 10^{4}$ cells/well and were preincubated at $37^{\circ} \mathrm{C}$ in a humidified atmosphere containing $5 \% \mathrm{CO}_{2}$. Subsequently, $10 \mu \mathrm{l}$ CCK-8 solution was added to each well, and the plates were incubated for $2 \mathrm{~h}$ in an incubator. The absorbance of each well was measured using a microplate reader at $450 \mathrm{~nm}$ (BioTek Instruments, Inc.) at room temperature $\left(25^{\circ} \mathrm{C}\right)$. Cell viability was calculated using the following equation: Viability $=\left(\mathrm{OD}_{\text {test group }}-\mathrm{OD}_{\text {blank group }}\right) /\left(\mathrm{OD}_{\text {control }}\right.$ group $\left.-\mathrm{OD}_{\text {blank group }}\right)$ x $100 \%$; where OD refers to optical density.

TdT-mediated dUTP nick end labeling (TUNEL) staining. According to the manufacturer's protocol, TUNEL staining was conducted using an in situ cell death detection kit (Roche Diagnostics GmbH, Mannheim, Germany) to detect apoptosis of HPMECs, as described previously (18). DAPI (Nanjing KeyGen Biotech Co., Ltd.) was used to stain nuclei. In situ apoptosis detection (Abcam, Cambridge, MA, USA) was used to assess the apoptotic rate of paraffin-embedded lung sections according to the manufacturer's protocols. DAB reacted with the horseradish peroxidase (HRP)-labeled sample to generate a dark brown signal at the site of DNA fragmentation. TUNEL-positive cells were counted in 5 randomly selected fields (x400 magnification) under an inverted microscope (TE2000-U; Nikon Corporation).

Annexin V-FITC/propidium iodide (PI) staining. Following the indicated treatments, cells were collected and resuspended in $500 \mu \mathrm{l} 1 \mathrm{X}$ Annexin $\mathrm{V}$ binding buffer, after which they were incubated with $5 \mu \mathrm{l}$ Annexin V-FITC and $5 \mu \mathrm{l}$ PI for $15 \mathrm{~min}$ at room temperature in the dark. Flow cytometry (FCM; BD Biosciences, Franklin Lakes, NJ, USA) was performed to detect apoptotic cells.

Western blot analysis. Radioimmunoprecipitation assay buffer was used to extract total protein, and aMembrane and Cytoplasmic Protein Extraction kit (both Nanjing KeyGen Biotech Co., Ltd.) was used to extract protein from cells and the left lung tissues of mice from each treatment group according to the manufacturer's protocols. Bicinchoninic acid kit was used to measure protein concentration. Equivalent amounts of protein $(30 \mu \mathrm{g})$ were separated by SDS-PAGE and were electrotransferred to polyvinylidene fluoride membranes. Subsequently, the membranes were blocked with $5 \%$ dry milk/bovine serum albumin (BSA) at room temperature $\left(25^{\circ} \mathrm{C}\right)$ for $1 \mathrm{~h}$, and were immunoblotted with

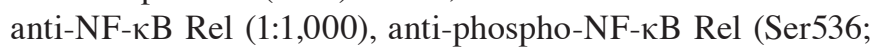
$1: 1,000)$, anti- $\beta$-catenin $(1: 500)$, anti-VE-cadherin $(1: 1,000)$, anti-NADPH oxidase antibody (1:500), anti-GSK-3 $\beta$ (1:500), anti-phospho-GSK-3 $\beta$ (Ser9; 1:500) and anti-GAPDH $(1: 8,000)$ primary antibodies overnight at $4^{\circ} \mathrm{C}$, followed by incubation with the corresponding HRP-conjugated secondary antibodies (1:5,000). Protein bands were detected according to an enhanced chemiluminescence (ECL) method (EMD Millipore, Billerica, MA, USA) using a Bio-Rad Gel Imaging system and were analyzed with Quantity One software version 4.4.0 (both Bio-Rad Laboratories, Inc., Hercules, CA, USA). The expression levels were determined by measuring the corresponding band intensities.

Reverse transcription-quantitative polymerase chain reaction $(R T-q P C R)$. Total RNA was isolated from cells using TRIzol reagent (Invitrogen; Thermo Fisher Scientific, Inc., Waltham, MA, USA) according to the manufacturer's protocol. The relative RNA levels were quantified using a NanoDrop 2000 spectrophotometer (NanoDrop; Thermo Fisher Scientific, Wilmington, DE, USA). Subsequently, $1 \mu \mathrm{g}$ RNA was used as a template for the generation of cDNA using a HiScript First Strand cDNA synthesis kit (Vazyme, Piscataway, NJ, USA). The reverse transcription reaction conditions were $25^{\circ} \mathrm{C}$ for $5 \mathrm{~min}, 42^{\circ} \mathrm{C}$ for $15 \mathrm{~min}$, and $85^{\circ} \mathrm{C}$ for $5 \mathrm{~min}$. Gene expression levels of TNF- $\alpha$, IL- 6 , VE-cadherin and $\beta$-catenin were detected using a HiScript ${ }^{\circledR}$ II One Step qRT-PCR SYBR $^{\circledR}$-Green kit (Vazyme) and StepOne Real-Time PCR apparatus (Applied Biosystem; Thermo Fisher Scientific, Inc., Waltham, MA, USA). Polymerase chain reaction conditions were as follows: pre-denaturation at $95^{\circ} \mathrm{C}$ for $30 \mathrm{sec}, 40$ cycles of denaturation at $95^{\circ} \mathrm{C}$ for $5 \mathrm{sec}$, annealing at $55^{\circ} \mathrm{C}$ for $30 \mathrm{sec}$, and polymerization at $72^{\circ} \mathrm{C}$ for $30 \mathrm{sec}$. Relative gene expression levels were normalized to GAPDH using CFX manager softwareversion 3.0 (Bio-Rad Laboratories, Inc.) according to the comparative $\mathrm{Cq}(\Delta \Delta \mathrm{Cq})$ method (31). The primer sequences are presented in Table I.

Immunofluorescence staining. Coverslips (3 from each group) of the HPMECs $\left(1 \times 10^{5}\right.$ cells per well) were fixed 
with $3.7 \%$ paraformaldehyde, permeabilized with $0.5 \%$ Triton X-100, blocked with PBS containing 5\% goat serum and incubated with anti-NF- $\kappa \mathrm{B}$ p65 antibodies $(1: 400)$ at $4^{\circ} \mathrm{C}$ overnight. Subsequently, coverslips were incubated with Alexa Fluor 488-labeled secondary antibodies (1:500) for $1 \mathrm{~h}$ in the dark. Coverslips were rinsed three times with PBS, and the nuclei were stained with DAPI (Nanjing KeyGen Biotech Co., Ltd.) for $5 \mathrm{~min}$. Images were captured by inverted microscopy (TE2000-U; Nikon Corporation) after washing.

Fluorescent phalloidin for F-actin staining. HPMECs (1x10 $10^{5}$ cells per well) were seeded on coverslips ( 3 from each group) and were fixed in $3.7 \%$ paraformaldehyde in PBS. The coverslips were then rinsed with PBS, permeabilized with $0.1 \%$ Triton X-100 in PBS, preincubated with PBS containing $1 \%$ BSA and stained with $200 \mu 1$ Fluorescent-iFluor ${ }^{\mathrm{TM}} 594$ phalloidin solution (Nanjing KeyGen Biotech Co., Ltd.) for $30 \mathrm{~min}$ at room temperature. Finally, coverslips were washed, sealed and images were captured by inverted microscopy (TE2000-U; Nikon Corporation).

Detection of ROS production. Intracellular ROS production was detected using the superoxide indicator dihydroethidium (DHE; D1168; Invitrogen; Thermo Fisher Scientific, Inc.). HPMECs were pretreated with rh-vaspin $(10 \mathrm{ng} / \mathrm{ml})$ or PBS as a control for $24 \mathrm{~h}$, and were then exposed to PBS or LPS (100 ng/ml) for $4 \mathrm{~h}$. HPMECs were washed twice with PBS, incubated in fresh culture medium without FBS, and were incubated with DHE (10 $\mu \mathrm{M}$ dissolved in dimethyl sulfoxide) for $15 \mathrm{~min}$ at $37^{\circ} \mathrm{C}$. Once the DHE probe is oxidized by ROS to 2-hydroxyethidium, it intercalates within DNA, resulting in the fluorescent red staining of cell nuclei. Images of the cells were captured using an inverted fluorescence microscope (TE2000-U; Nikon Corporation) from three different fields of view. Fluorescence intensity was measured using ImageJ software (National Institutes of Health, Bethesda, MD, USA), averages were calculated and were normalized to the control.

Lucigenin assay. Following exposure of HPMECs to PBS or LPS $(100 \mathrm{ng} / \mathrm{ml})$ for $4 \mathrm{~h}$ in the absence or presence of rh-vaspin $(100 \mathrm{ng} / \mathrm{ml})$ for $24 \mathrm{~h}$, total cell lysates were harvested. Nicotinamide adenine dinucleotide phosphate (NADPH) oxidase (NOX) activity was determined using a lucigenin assay. As a chemiluminescent probe, lucigenin is used to indicate the presence of superoxide anion radicals in cells. The reaction was performed in a total volume of $200 \mu \mathrm{l}$ assay buffer containing $10 \mu \mathrm{M}$ lucigenin (L-6868; NSC-151912; MedChem Express, Monmouth Junction, NJ, USA), $1 \mathrm{mM}$ NADPH, and $15 \mu \mathrm{g}$ cell lysates. Following incubation for $30 \mathrm{~min}$ at $37^{\circ} \mathrm{C}$ in the dark, absorbance was measured using a luminometer (BioTek Instruments, Inc.) at an excitation wavelength of $540 \mathrm{~nm}$ and an emission wavelength of $605 \mathrm{~nm}$. Samples were well mixed and chemiluminescence was continuously measured for $30 \mathrm{~min}$. Chemiluminescence of relative light units per second (RLU/sec) was obtained every $10 \mathrm{sec}$ and the results were calculated as area under the curve and normalized to the control.

Statistical analysis. Data are presented as the mean \pm standard deviation. Results are representative of at least three independent experiments performed in triplicate. Unpaired
Student's t-test was performed for comparisons between two independent groups. One-way analysis of variance (ANOVA) followed by the Student-Newman-Keuls post hoc test was performed to compare continuous variables with normal distribution from three or more independent groups, and to detect significant differences between particular groups. Kruskal-Wallis ANOVA followed by Mann-Whitney U test was performed to compare continuous variables with abnormal distribution from three or more independent groups, and to detect significant differences between particular groups. $\mathrm{P}<0.05$ (95\% confidence interval) was considered to indicate a statistically significant difference at. All statistical analyses were conducted using GraphPad Prism 5.0 (GraphPad Software, Inc., La Jolla, CA, USA).

\section{Results}

Vaspin attenuates LPS-induced lung injury and pulmonary inflammation in vivo and in vitro. In order to explore the effects of vaspin on lung histological injury in an LPS-induced mouse model of ARDS, lung histopathological examination was conducted. Mice were systemically instilled with Ad-vaspin or Ad- $\beta$-gal as control ( $3 \times 10^{7} \mathrm{PFU} /$ mouse for 3 days) and were subjected to intratracheal injection with LPS ( $5 \mathrm{mg} / \mathrm{kg})$ to establish a mouse model of ARDS or with PBS as a control. Plasma vaspin levels were enhanced to $99.0 \pm 7.9 \mathrm{ng} / \mathrm{ml}$ on day 3 after Ad-vaspin injection, which could not be detected in the control mice treated with Ad- $\beta$-gal (data not shown). Mice that were injected with LPS developed ARDS as early as $4 \mathrm{~h}$ after LPS insult, as indicated by histopathological alterations in the lungs, including increased inflammatory cell infiltration, thickened alveolar septum, intra-alveolar and interstitial edema fluid, and patchy areas of hemorrhage (Fig. 1A). These histopathological alterations were significantly attenuated following administration of Ad-vaspin (Fig. 1A and B). Since lung injury in ARDS is associated with an exaggerated inflammatory response, the present study further examined the effects of vaspin on pulmonary inflammation. Consistent with the results of lung injury assessment, expression levels of the proinflammatory cytokines TNF- $\alpha$ (Fig. 1C) and IL-6 (Fig. 1D) were reduced in Ad-vaspin-pretreatedmice compared with in Ad- $\beta$-gal-pretreated mice $4 \mathrm{~h}$ post-LPS insult, whereas expression of the anti-inflammatory cytokine IL-10 (Fig. 1E) was increased. In addition, the expression levels of the adhesion molecule, ICAM-1 (Fig. 1F), were reduced in Ad-vaspin-pretreated mice compared with in Ad- $\beta$-gal-pretreated mice $4 \mathrm{~h}$ post-LPS insult. These findings suggested that vaspin may exert an anti-inflammatory effect on lung injury in a mouse model of LPS-induced ARDS.

Vaspin has previously been reported to exert anti-inflammatory effects on various types of vascular ECs (24-27). Therefore, it may be hypothesized that vaspin mediates mitigation of inflammation in pulmonary ECs. To assess the anti-inflammatory effects of vaspin in vitro, the mRNA expression levels of inflammatory cytokines (TNF- $\alpha$ and IL-6) and endothelial-specific adhesion markers (VCAM-1 and E-selectin) were analyzed in HPMECs $2 \mathrm{~h}$ after LPS insult. Consistent with the LPS-mediated inflammatory response in lung tissue, the expression levels of TNF- $\alpha$ (Fig. 2A), IL-6 (Fig. 2B), VCAM-1 (Fig. 2C) and E-selectin (Fig. 2D) were significantly increased in HPMECs following LPS 
A
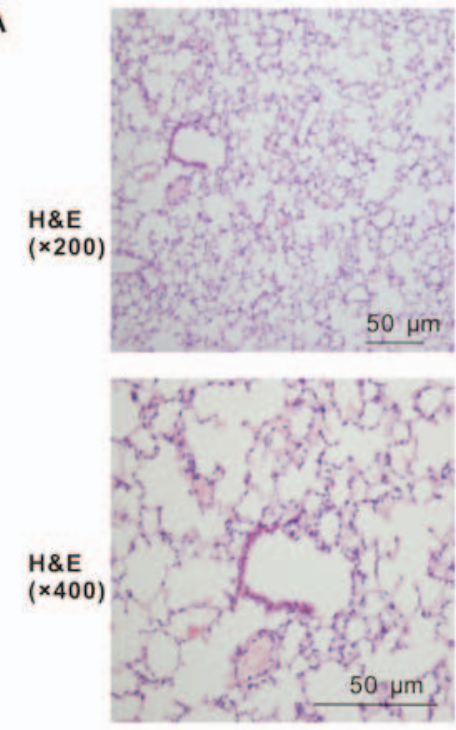

Ad- $\beta$-gal

Ad-vaspin

B

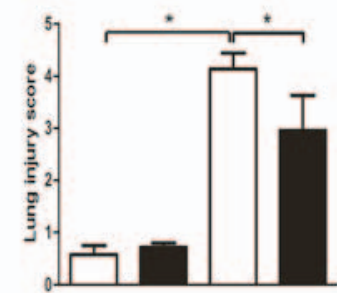

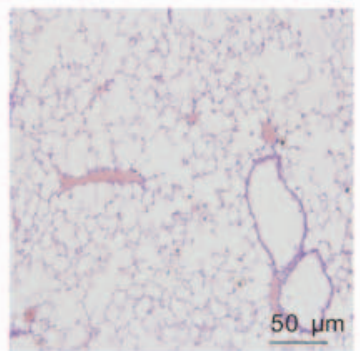

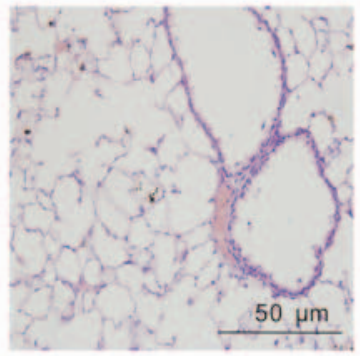

$+$

control

C

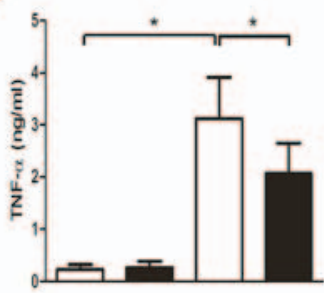

D
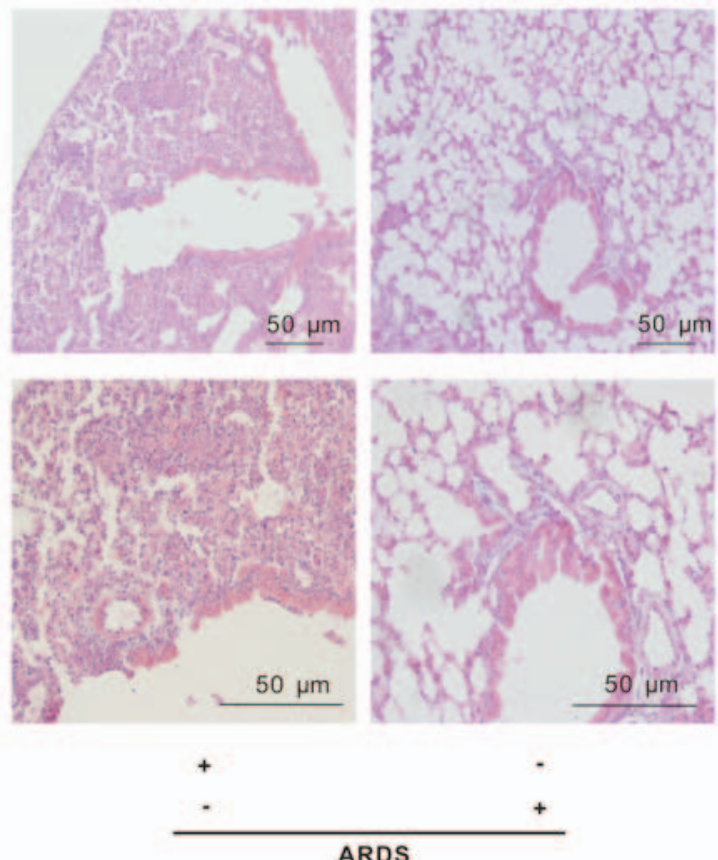

ARDS

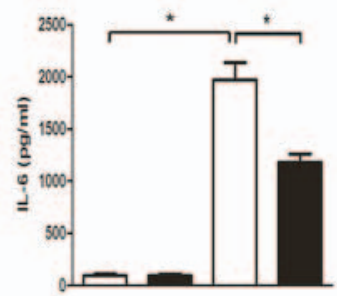

E

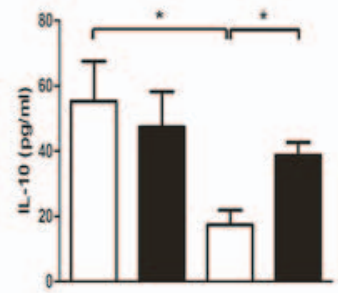

Ad- $\beta$-gal + - + -

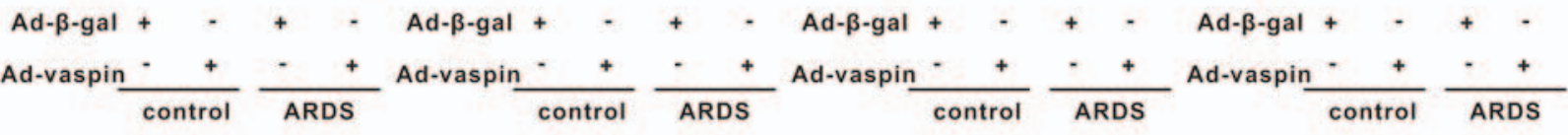

$\mathrm{F}$
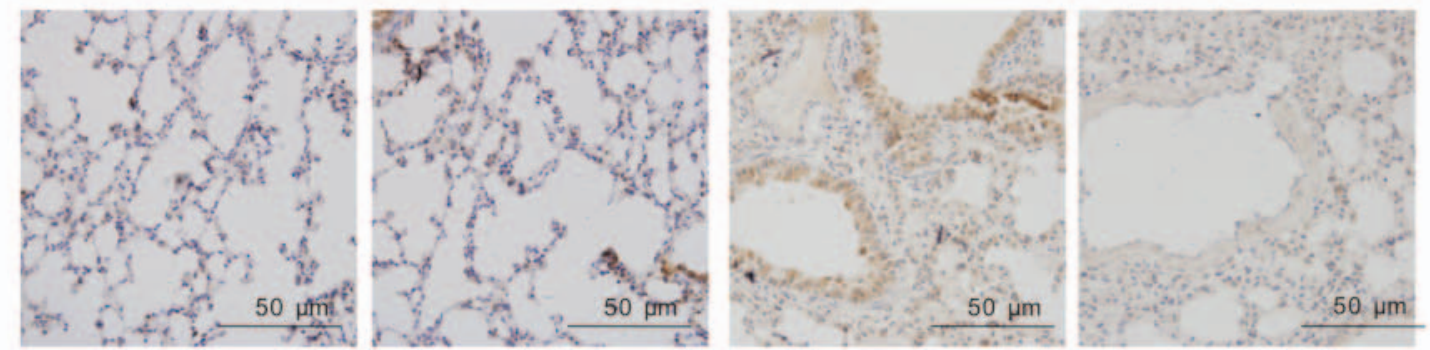

Ad- $\beta$-gal

Ad-vaspin
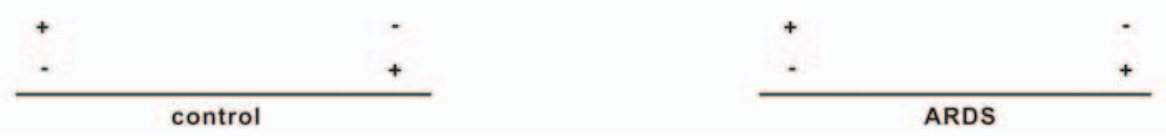

Figure 1. Vaspin ameliorates LPS-induced lung injury and pulmonary inflammation in vivo. Mice were systemically instilled with Ad-vaspin or Ad- $\beta$-gal as a control ( $3 \times 10^{7} \mathrm{PFU} /$ mouse) for 3 days and were subjected to intratracheal injection with LPS $(5 \mathrm{mg} / \mathrm{kg})$ in order to establish a mouse model of ARDS. Mice were intratracheally injected with PBS as a control. A total of $4 \mathrm{~h}$ after LPS/PBS injection, lung lobes were harvested. (A) Lung hispathological alterations were assessed by hematoxylin and eosin staining ( $\mathrm{n}=5$ mice from each group assessed in triplicate; magnification, $\mathrm{x} 200$ and $\mathrm{x} 400$ ). (B) Lung injury scores were used to analyze lung histopathological alterations ( $n=5$ mice from each group assessed in triplicate). Expression levels of (C) TNF- $\alpha$, (D) IL-6 and (E) IL-10 were measured in lung homogenates by ELISA ( $\mathrm{n}=5$ mice from each group assessed in triplicate). (F) Expression levels of ICAM-1 were detected by immunohistochemistry ( $\mathrm{n}=5$ mice from each group assessed in triplicate; magnification, $\mathrm{x} 400$ ). Data are presented as the mean \pm standard deviation. ${ }^{*} \mathrm{P}<0.05$. Ad- $\beta$-gal, adenoviral vector expressing $\beta$-galactosidase; Ad-vaspin, adenoviral vector expressing vaspin; ARDS, acute respiratory distress syndrome; ICAM-1, intercellular adhesion molecule-1; IL, interleukin; LPS, lipopolysaccharide; TNF- $\alpha$, tumor necrosis factor- $\alpha$.

administration, which were reversed by pretreatment with rh-vaspin. The observed anti-inflammatory effects of vaspin were further confirmed by examining the activation of NF- $\kappa B$, which is a pivotal inflammatory mediator; the results indicated that the phosphorylation and nuclear translocation of the NF- $\mathrm{KB}$ Rel subunit were reduced following treatment of HPMECs with rh-vaspin $2 \mathrm{~h}$ after LPS insult (Fig. 2E and F). Taken together, these findings indicated that vaspin may exert a protective role during the early stage of LPS-induced ARDS via the suppression of EC inflammation. 

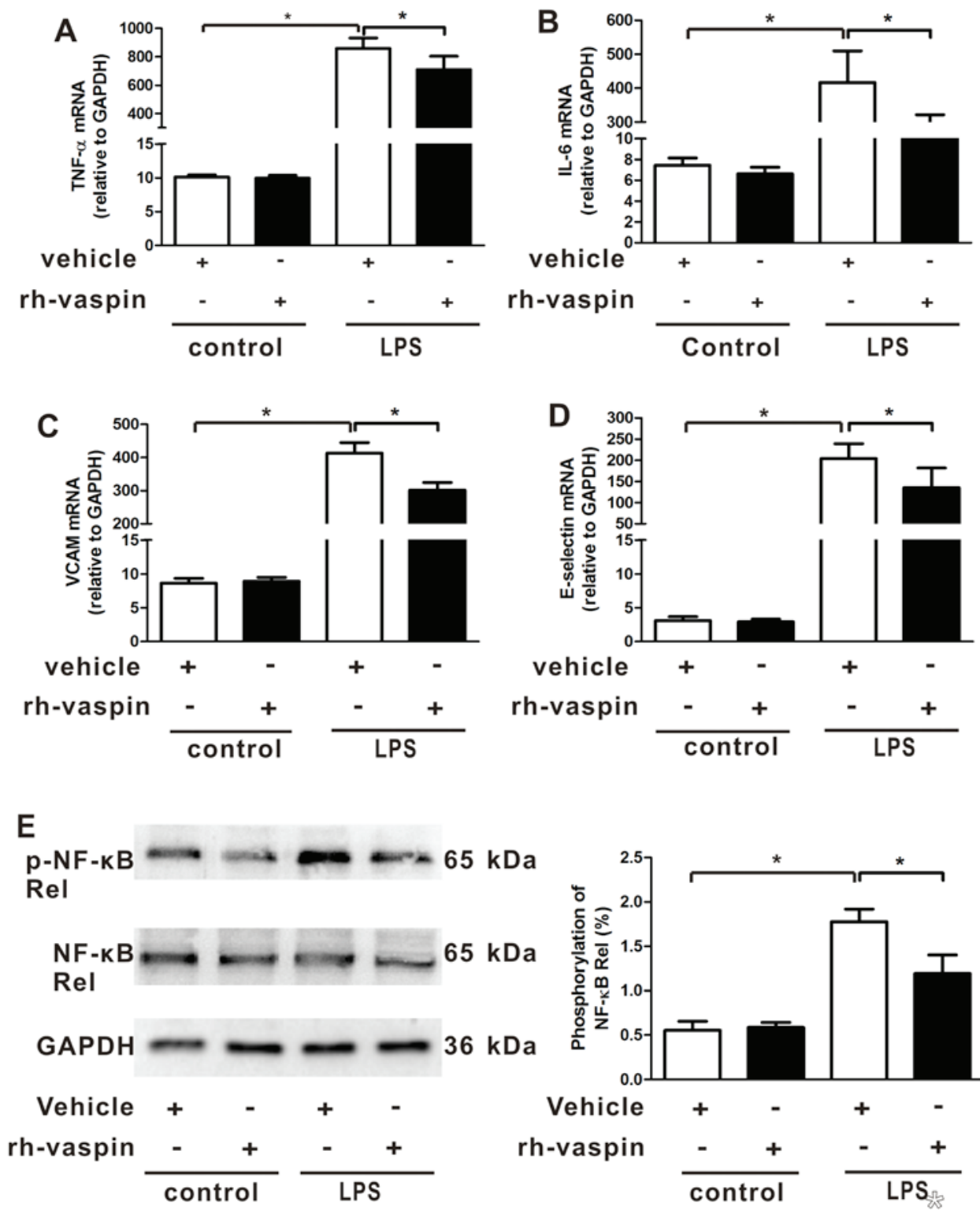
$65 \mathrm{kDa}$ $36 \mathrm{kDa}$

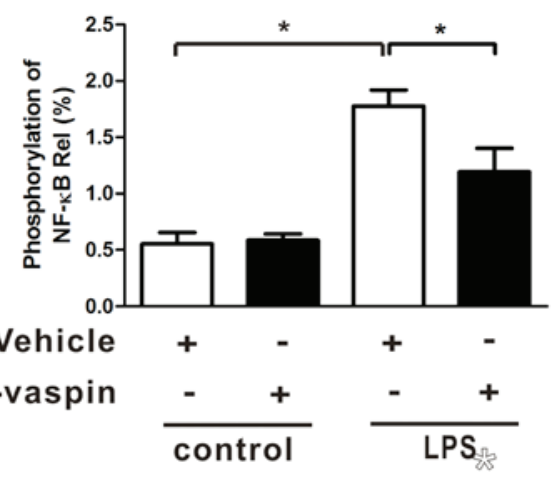

$\mathbf{F}$
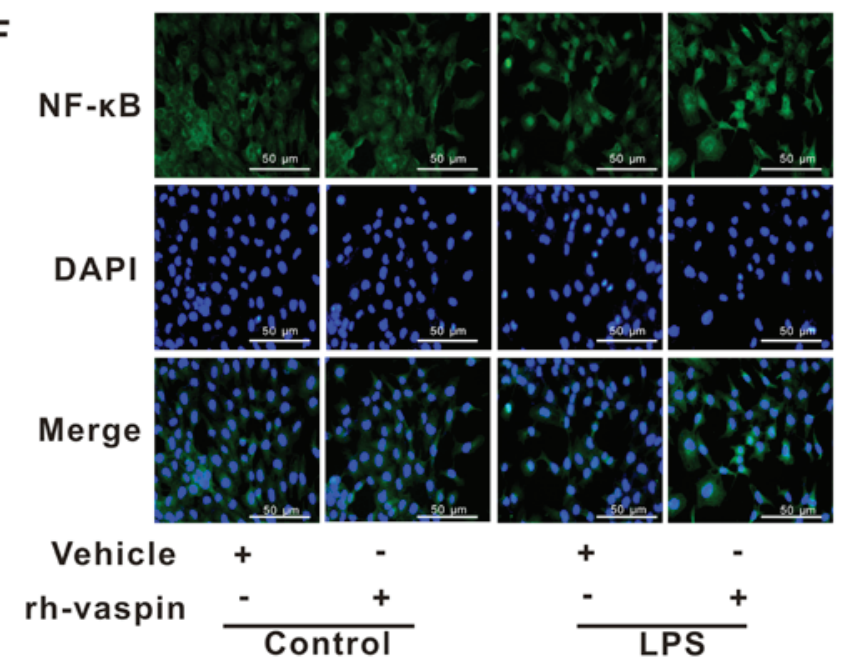

Figure 2. Vaspin attenuates LPS-induced endothelial cell inflammation in vitro. HPMECs were pretreated with rh-vaspin (10 ng/ml) or PBS as a control for $24 \mathrm{~h}$, and were then exposed to LPS (100 ng/ml) or vehicle (PBS) for $2 \mathrm{~h}$. mRNA expression levels of (A) TNF- $\alpha$, (B) IL-6, (C) VCAM-1 and (D) E-selectin in the HPMECs lysate were assessed by quantitative polymerase chain reaction ( $\mathrm{n}=5$ cultures from each group assessed in triplicate). (E) Expression levels of

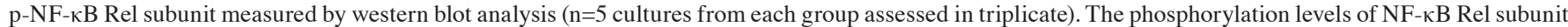

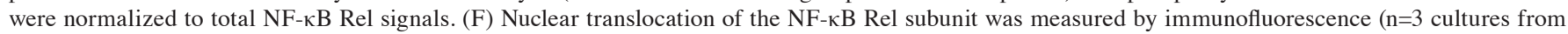
each group assessed in triplicate). Data are presented as the mean \pm standard deviation. ${ }^{*} \mathrm{P}<0.05$. HPMECs, human pulmonary microvascular endothelial cells;

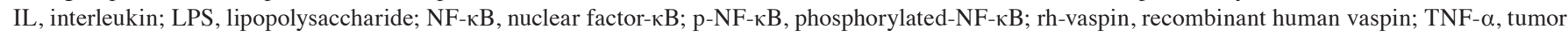
necrosis factor- $\alpha$; VCAM-1, vascular cell adhesion molecule 1. 


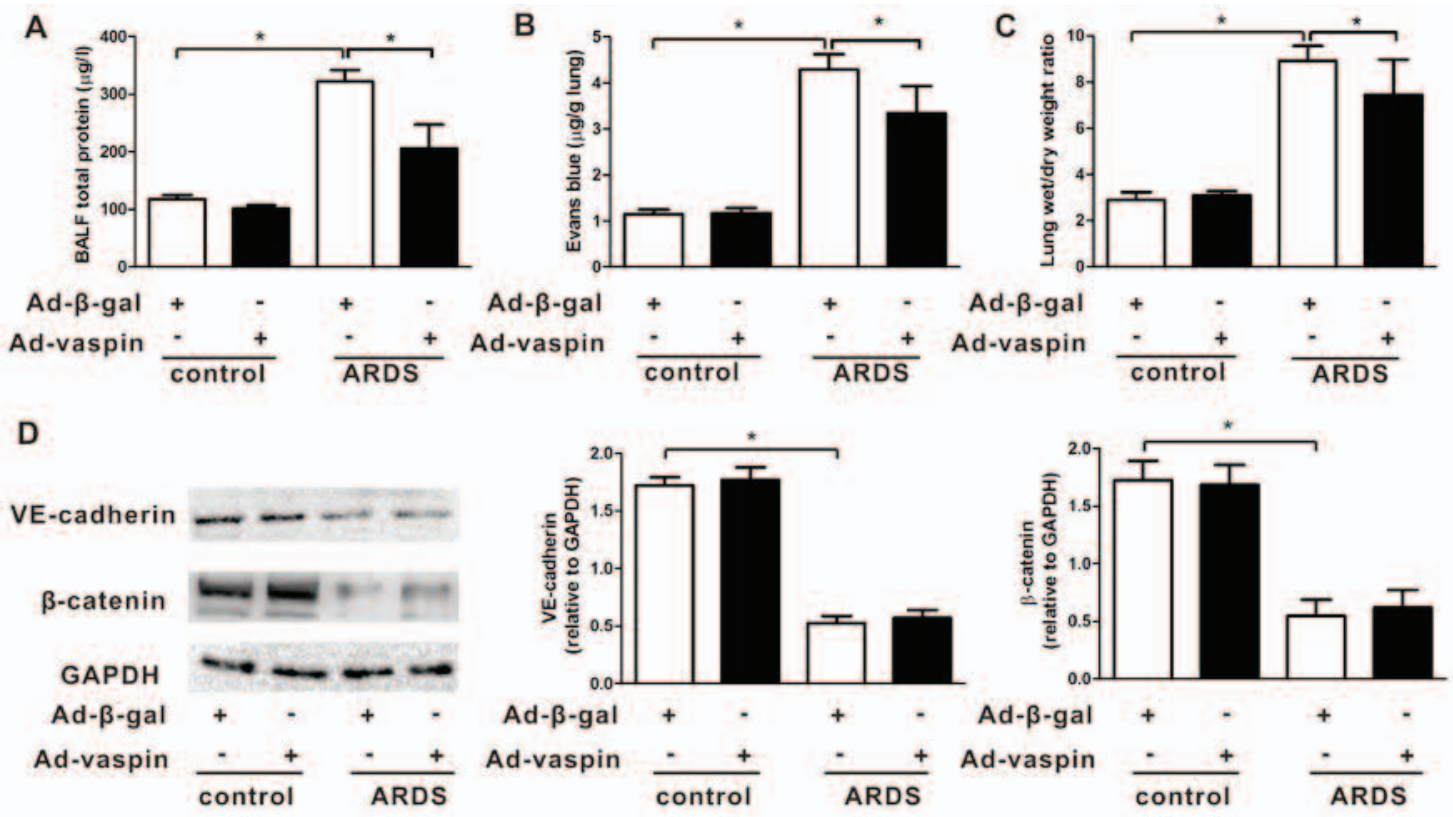

$\mathrm{E}$

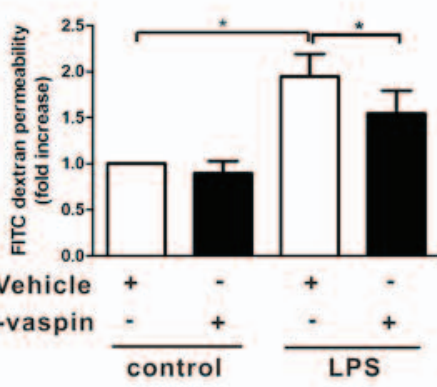

H
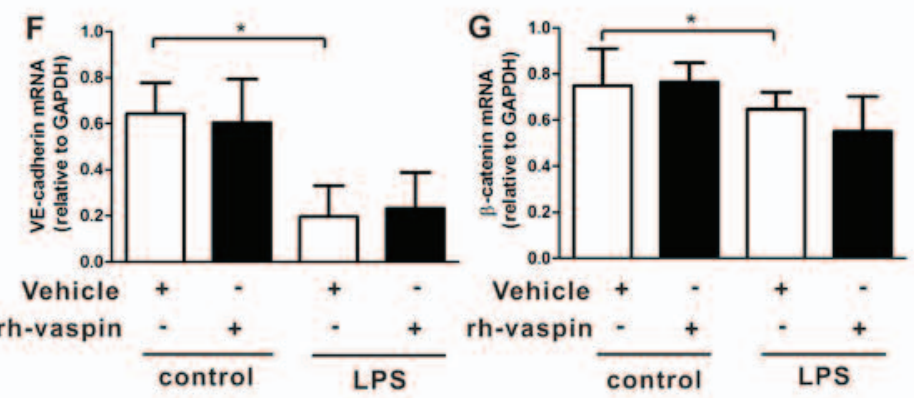
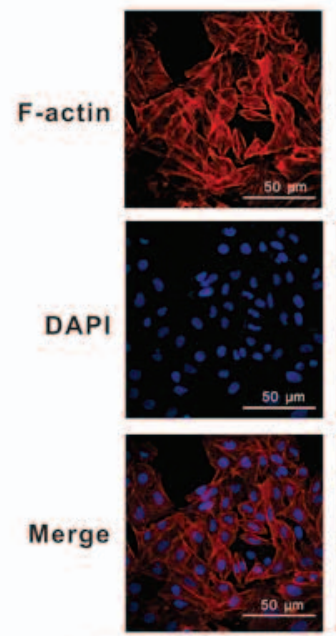

Vehicle
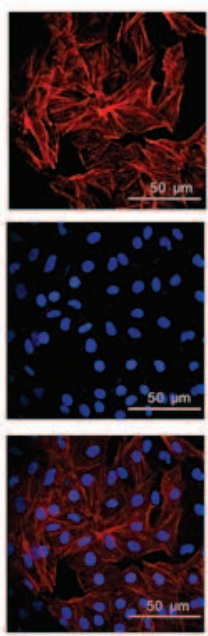

rh-vaspin

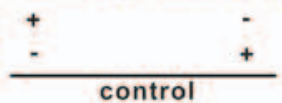

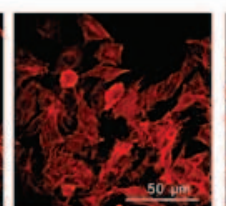
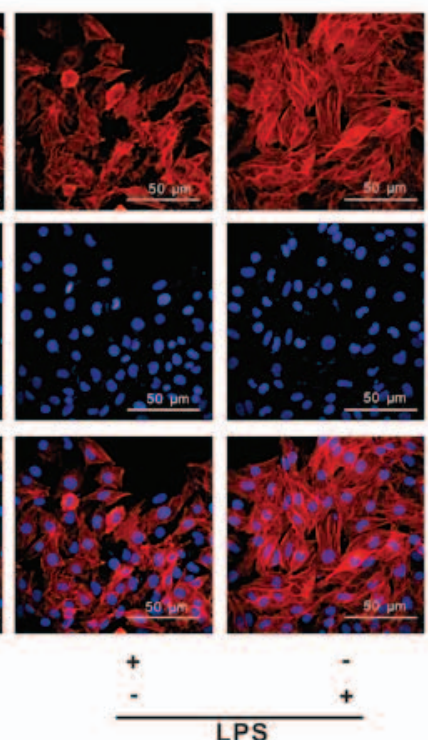

Figure 3. Vaspin reverses pulmonary endothelial cell barrier dysfunction in vivo and in vitro, with no effect on pulmonary endothelial cell adherens junctions and the actin cytoskeleton following LPS insult. Mice were systemically instilled with Ad-vaspin or Ad- $\beta$-gal as a control (3x $10^{7} \mathrm{PFU} / \mathrm{mouse}, 3$ days) and were subjected to an intratracheal injection with LPS (5 mg/kg) to establish a mouse model of ARDS. Mice were intratracheally injected with PBS as a control. (A) Total BALF protein concentrations, (B) Evans blue dyed-albumin extravasation and (C) wet/dry ratios in a murine model of ARDS were measured $4 \mathrm{~h}$ post-LPS injection ( $\mathrm{n}=5$ mice from each group assessed in triplicate). (D) Expression levels of VE-cadherin and $\beta$-catenin were detected in lung tissues by western blot analysis ( $\mathrm{n}=5$ mice from each group assessed in triplicate). Relative protein expression levels were semi-quantified by measuring corresponding band intensities, and values were expressed relative to GADPH. HPMECs were pretreated with rh-vaspin $(10 \mathrm{ng} / \mathrm{ml})$ or PBS as a control for $24 \mathrm{~h}$, and were then exposed to PBS or LPS $(100 \mathrm{ng} / \mathrm{ml})$ for $4 \mathrm{~h}$. (E) Influx of FITC-dextran was measured by permeability assay ( $\mathrm{n}=5$ cultures from each group assessed in triplicate). mRNA expression levels of (F) VE-cadherin and (G) $\beta$-catenin in the HPMECs lysate were assessed by quantitative polymerase chain reaction (n=5 cultures from each group assessed in triplicate). $(\mathrm{H})$ Actin cytoskeleton distribution was assessed by phalloidin staining ( $\mathrm{n}=3$ cultures from each group assessed in triplicate). Data are presented as the mean \pm standard deviation. "P $<0.05$. Ad- $\beta$-gal, adenoviral vector expressing $\beta$-galactosidase; Ad-vaspin, adenoviral vector expressing vaspin; ARDS, acute respiratory distress syndrome; BALF, bronchoalveolar lavage fluid; FITC, fluorescein isothiocyanate; HPMECs, human pulmonary microvascular endothelial cells; LPS, lipopolysaccharide; rh-vaspin, recombinant human vaspin; VE-cadherin, vascular endothelial-cadherin. 
Vaspin restores the pulmonary EC barrier following LPS insult in vivo and in vitro. Pulmonary microvascular hyperpermeability is a common feature of ARDS; therefore, it was assessed in vitro and in vivo in the present study (Fig. 3). To address whether vaspin modulates pulmonary endothelial barrier function in vivo, total bronchoalveolar lavage fluid (BALF) protein concentrations, EBDA extravasation and lung W/D ratios were analyzed in mice with LPS-induced ARDS. Lung histological damage was observed to be associated with pulmonary microvascular hyperpermeability following LPS instillation, as manifested by increases in BALF protein concentrations (Fig. 3A), EBDA extravasation (Fig. 3B) and W/D ratios (Fig. 3C) at $4 \mathrm{~h}$ after LPS treatment. The time point selected to evaluate the effects of vaspin on ARDS was based on its coincidence with the histological onset of lung injury in mice. Pretreatment with Ad-vaspin significantly attenuated pulmonary microvascular hyperpermeability in a murine model of ARDS (Fig. 3A-C). In addition, to further confirm the ability of vaspin to mitigate LPS-induced EC hyperpermeability in vitro, HPMECs were cultured in the presence or absence of rh-vaspin $(10 \mathrm{ng} / \mathrm{ml})$ for $24 \mathrm{~h}$, and the influx of FITC-dextran was measured. Treatment with a physiological concentration of rh-vaspin prevented LPS-induced increases in the influx of FITC-dextran (Fig. 3E). These data indicated that vaspin may significantly attenuate LPS-challenged pulmonary microvascular hyperpermeability in vivo and EC barrier dysfunction in vitro.

Vaspin has no effect on pulmonary EC adherens junctions $(A J s)$, actin cytoskeleton and EC differentiation following LPS insult in vivo and in vitro. The stabilization of interendothelial AJs and the actin cytoskeleton are essential for a restrictive pulmonary EC barrier; lung endothelium permeability can increase due to alterations in AJs and the endothelial cytoskeleton. Therefore, to further address the effects of vaspin on vascular homeostasis, the expression levels of VE-cadherin and $\beta$-catenin, which are two important AJ proteins of ECs, were evaluated in the lung tissue of mice. Notably, analysis of AJs expression did not detect a difference in the Ad-vaspin-pretreated mice compared with those treated with LPS alone (Fig. 3D). In addition, LPS caused a reduction in the mRNA expression levels of AJs (Fig. 3F and G), as well as cell retraction, F-actin reorganization and stress fiber formation in HPMECs, as determined by phalloidin staining (Fig. 3H). However, consistent with the results of the in vivo experiments, AJs gene expression and distribution of the actin cytoskeleton in vaspin-treated HPMECs were not altered compared with the controls treated with LPS alone (Fig. 3F-H). Collectively, these findings indicated that vaspin may exert beneficial effects on pulmonary microvascular hyperpermeability via restoring EC barrier function; however, these effects may not depend on the ability of vaspin to regulate interendothelial AJs or the endothelial cytoskeleton but may be dependent on other mechanisms associated with endothelial barrier integrity.

Vaspin improves pulmonary EC survival and suppresses pulmonary EC apoptosis following LPS insult in vitro and in vivo. Vaspin has been reported to inhibit the apoptosis of ECs, including human aortic ECs, HUVECs and smooth muscle cells (SMCs). Furthermore, the function of the pulmonary endothelial barrier depends on its integrity $(24,25,27)$. Focusing on the beneficial effects of vaspin on the function and integrity of pulmonary ECs at the cellular level, HPMECs were treated with LPS in the presence or absence of rh-vaspin (10 ng/ml, pretreatment for $24 \mathrm{~h}$ ). CCK-8, TUNEL staining and FCM analyses were performed to ascertain whether vaspin modulates the survival and apoptosis of pulmonary ECs following exposure to LPS (Fig. 4). In HPMECs, pretreatment with rh-vaspin significantly promoted pulmonary EC survival and suppressed pulmonary EC apoptosis under LPS stimulus compared with those pretreated with PBS, as determined by increased cell viability (Fig. 4B), a reduction in the number of TUNEL-positive cells (Fig. 4A and C) and reduced apoptotic rate, as determined by FCM (Fig. 4A and D). In situ apoptotic detection was further performed to verify the effects of vaspin on apoptosis in lung tissues. Compared with in the control group, Ad-vaspin markedly reduced the number of TUNEL-positive cells in lung tissues post-LPS insult (Fig. 4E). These results suggested that vaspin exerts a protective role on HPMECs, at least partially via its prosurvival and anti-apoptotic properties.

Vaspin inhibits LPS-induced ROS generation and NOX activation following LPS insult in vitro. A previous study demonstrated that ROS and NOX mediated EC apoptosis under various insults. Furthermore, vaspin has been demonstrated to inhibit ROS generation by suppressing NOX activation in ECs, including HUVECs. Therefore, the present study further evaluated the effects of vaspin on intracellular ROS production by measuring the fluorescence intensity of the intracellular fluorescent probe, DHE. Compared with HPMECs exposed to LPS alone, pretreatment with rh-vaspin significantly counteracted LPS-induced ROS production in HPMECs (Fig. 5A and B). Constitutive NOX functions as an oxygen sensor that regulates intracellular superoxide organization. Therefore, to elucidate the upstream mechanisms, the effects of vaspin on NOX were further assessed by measuring NOX activity and expression. The results indicated that LPS induced an increase in NOX activity (Fig. 5C) and expression (Fig. 5D and E); however, this was markedly abrogated by pretreatment of HPMECs with vaspin. These findings indicated that the protective effects of vaspin against LPS insults in ECs may be associated with antioxidative properties.

Vaspin activates the Akt-GSK3 $\beta$ signaling pathway in vivo and in vitro. The PI3K/Akt signaling pathway acts as a compensatory regulator of ARDS through its anti-inflammatory, anti-apoptotic and antioxidant effects in response to numerous growth factors. Therefore, to assess the effects of vaspin on the activation of Akt-related signaling in vivo and in vitro, the phosphorylation of Akt and its downstream target GSK $3 \beta$ were assessed by western blotting. The results indicated that p-Akt and p-GSK3 $\beta$ levels were low under non-stressed conditions but were increased in a mouse model of LPS-induced ARDS; however, the differences between these groups were not statistically significant, thus suggesting an endogenous negative feedback mechanism underlying the effects of LPS on the PI3K/Akt pathway. Notably, administration of vaspin enhanced the phosphorylation of Akt and GSK3 $\beta$ in mouse lungs subjected to LPS (Fig. 6A). At the 
A
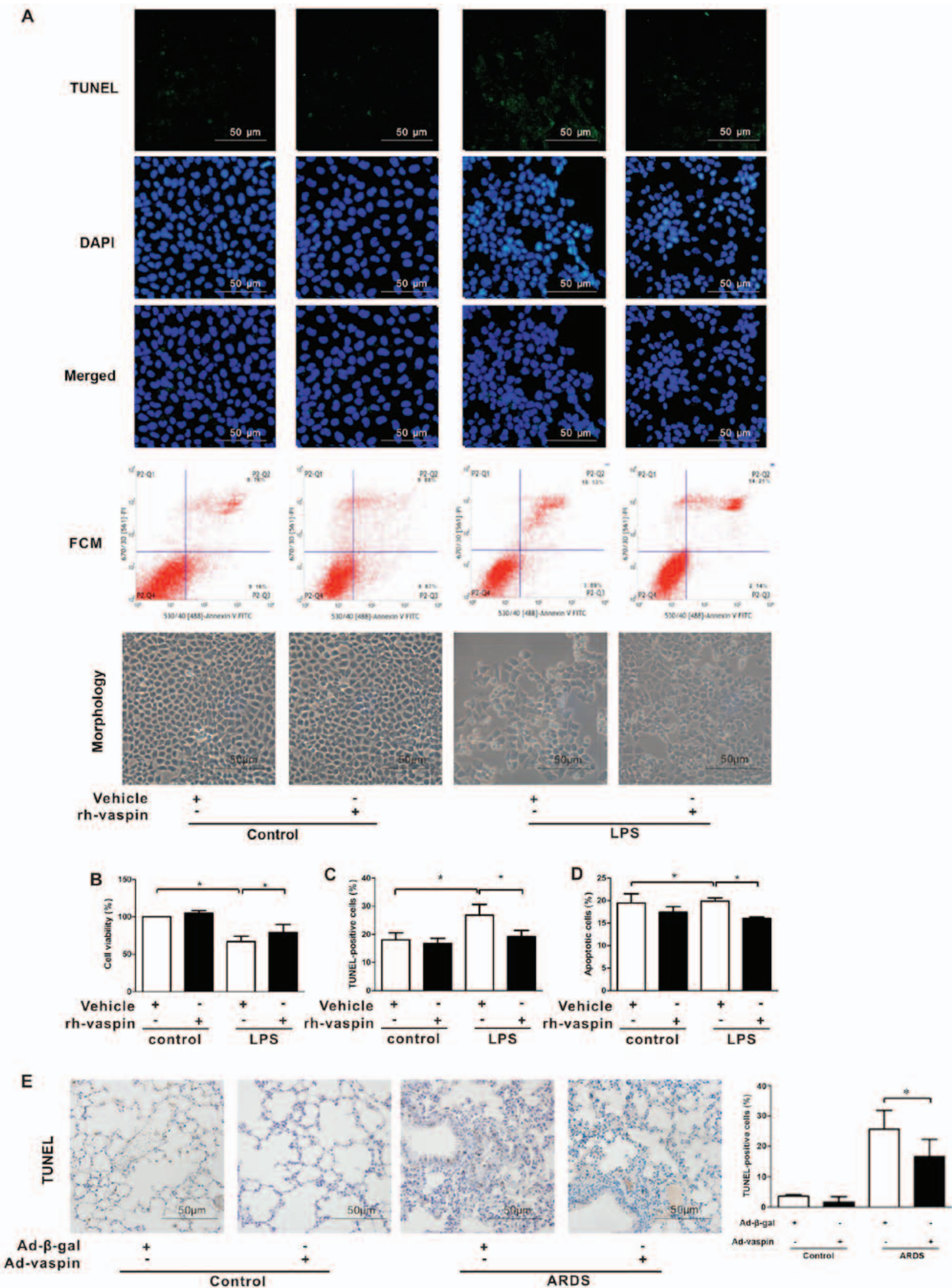

Figure 4. Vaspin improves survival and suppresses apoptosis following LPS insult in vitro and in vivo. HPMECs were pretreated with rh-vaspin (10 ng/ml) or PBS as a control for $24 \mathrm{~h}$, and were then exposed to PBS or LPS $(100 \mathrm{ng} / \mathrm{ml}$ ) for $2 \mathrm{~h}$. (A) Representative images of TUNEL staining and FCM ( $\mathrm{n}=3$ cultures from each group assessed in triplicate). (B) HPMEC survival was measured by Cell Counting kit-8 analyses ( $\mathrm{n}=5$ cultures from each group assessed in triplicate). (C) Quantitative analysis of TUNEL-positive HPMECs. (D) Quantitative analysis of apoptotic HPMECs, as determined by FCM. (E) Left panel, representative images of TUNEL staining of lung tissues; right panel, quantitative analysis of the number of TUNEL-positive cells. Data are presented as the mean \pm standard deviation. ${ }^{*} \mathrm{P}<0.05$. Ad- $\beta$-gal, adenoviral vector expressing $\beta$-galactosidase; Ad-vaspin, adenoviral vector expressing vaspin; FCM, flow cytometry; HPMECs, human pulmonary microvascular endothelial cells; LPS, lipopolysaccharide; rh-vaspin, recombinant human vaspin; TUNEL, TdT-mediated dUTP nick end labeling. 


\section{A}
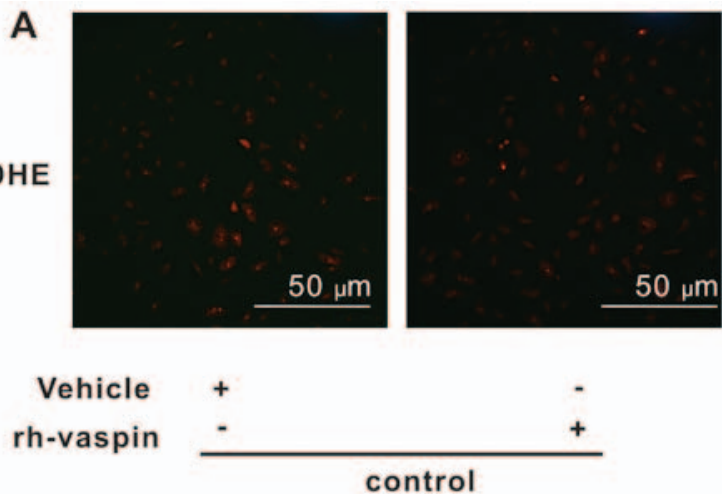

B

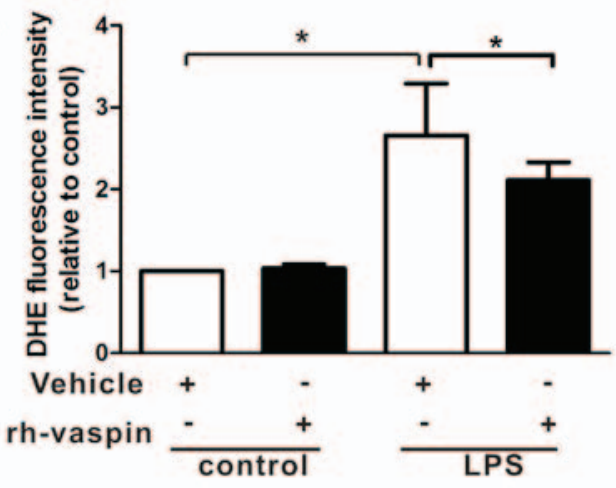

D

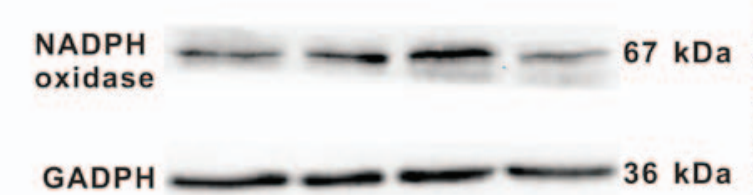

\begin{tabular}{rlr} 
Vehicle & + & - \\
rh-vaspin & - & + \\
\cline { 3 - 4 } & & control
\end{tabular}

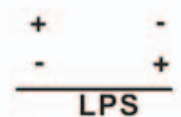

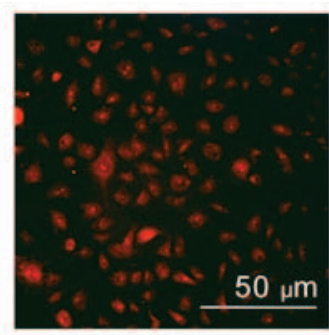
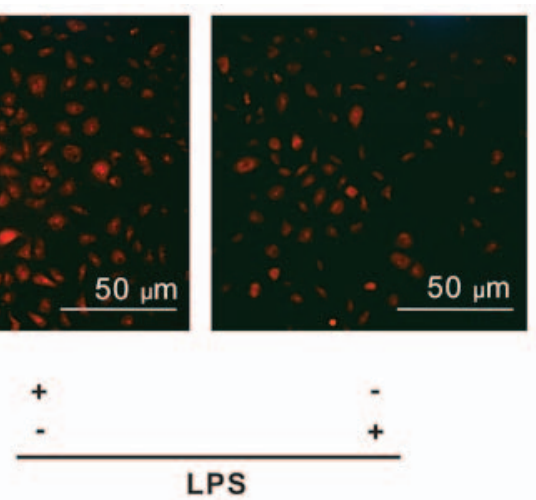

C

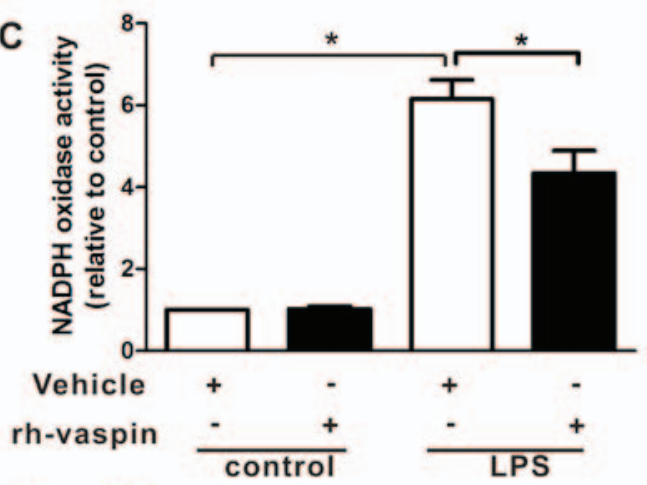

E

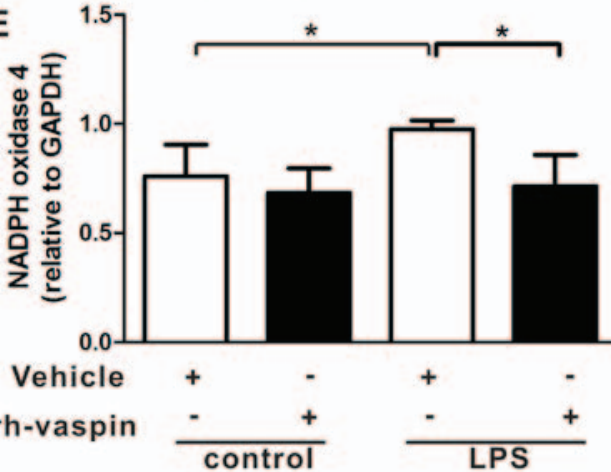

Figure 5. Vaspin inhibits LPS-induced ROS generation and NADPH oxidase activation following LPS insults in vitro. HPMECs were pretreated with rh-vaspin $(10 \mathrm{ng} / \mathrm{ml})$ or PBS as a control for $24 \mathrm{~h}$, and were then exposed to PBS or LPS (100 ng/ml) for $2 \mathrm{~h}$. (A) Representative images of DHE staining of HPMECs ( $\mathrm{n}=3$ cultures from each group assessed in triplicate). (B) Quantitative analysis of ROS production in HPMECs, as determined by fluorescence staining using DHE. (C) Quantitative analysis of NADPH oxidase activity in HPMECs, as determined by lucigenin assay. (D) Expression of NADPH oxidase was determined by western blot analysis. Relative protein expression levels were semi-quantified by measuring band intensities, and values were expressed relative to GADPH ( $\mathrm{n}=5$ cultures from each group assessed in triplicate). (E) Semi-quantitative analysis of NADPH oxidase expression in HPMECs, as determined by western blot analysis. Data are presented as the mean \pm standard deviation. *P<0.05. DHE, dihydroethidium; HPMECs, human pulmonary microvascular endothelial cells; LPS, lipopolysaccharide; NADPH, nicotinamide adenine dinucleotide phosphate; rh-vaspin, recombinant human vaspin; ROS, reactive oxygen species.

cellular level, rh-vaspin stimulated the phosphorylation of Akt and GSK3 $\beta$ (Fig. 6B). Collectively, these data suggested that vaspin acts as a stimulatory factor for the Akt-GSK3 $\beta$ signaling pathway, and vaspin-induced protection of pulmonary ECs during ARDS may be mediated, at least partially, by activating the Akt-GSK3 $\beta$ signaling pathway.

\section{Discussion}

Pulmonary vascular hyperpermeability has long been considered a principal pathological hallmark of ARDS, which is largely responsible for pulmonary edema formation $(3,4,32)$. Therefore, stability and integrity of the pulmonary endothelial barrier are important in the pathogenesis of ARDS. To the best of our knowledge, the present study is the first to provide evidence to suggest that vaspin protects the pulmonary endothelial barrier during LPS-induced ARDS by limiting inflammation, apoptosis and ROS generation in pulmonary ECs. In a murine model of ARDS, systemic administration of Ad-vaspin attenuated lung injuries by suppressing the pulmonary inflammatory response and restoring pulmonary endothelial barrier functions. In HPMECs, treatment with a physiological concentration of rh-vaspin led to attenuation of the inflammatory response, cell apoptosis and NOX-dependent ROS generation, as well as enhancement of EC survival, with no alterations in cell AJs expression or cytoskeletal rearrangement. A further mechanistic study suggested that the beneficial effects of vaspin are, at least partially, mediated by activation of the Akt-GSK3 $\beta$ signaling 
A

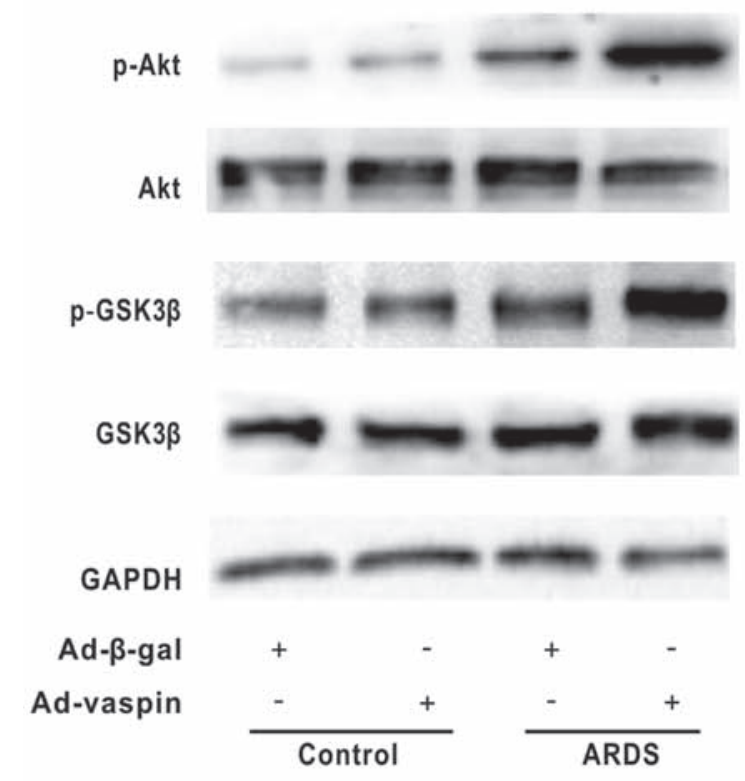

B

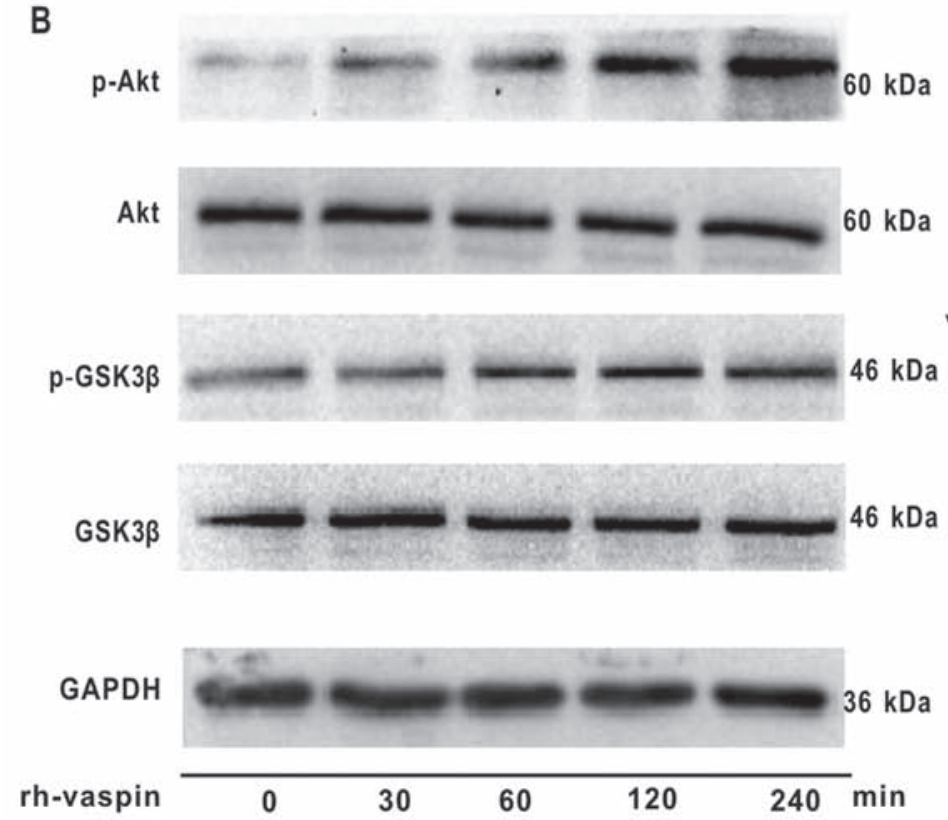

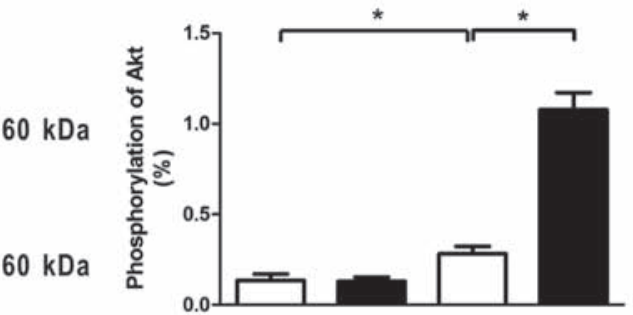

$46 \mathrm{kDa}$

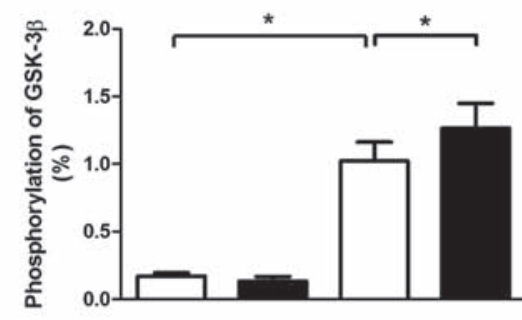

$\begin{array}{llllll}\text { Ad- } \beta \text {-gal } & + & - & + & + \\ \text { Ad-vaspin } & - & + & & - & + \\ & \text { Control } & & \text { ARDS }\end{array}$

Figure 6. Vaspin activates the Akt/GSK3 $\beta$ signaling pathways in vivo and in vitro. (A) Ad-vaspin enhanced phosphorylation of Akt and GSK3 $\beta$ in mouse lungs subjected to LPS. HPMECs were pretreated with rh-vaspin $(10 \mathrm{ng} / \mathrm{ml})$ or PBS as a control for $24 \mathrm{~h}$, and were then exposed to PBS or LPS (100 $\mathrm{ng} / \mathrm{ml})$ for $4 \mathrm{~h}$ ( $\mathrm{n}=5$ mice from each group assessed in triplicate). (B) Time-dependent alterations in the phosphorylation of Akt and GSK3 $\beta$ in HPMECs following rh-vaspin stimulation. Relative protein expression levels were semi-quantified by measuring corresponding band intensities; relative phosphorylation levels of p-Akt and p-GSK $3 \beta$ are normalized to total Akt and GSK3 $\beta$ ( $n=5$ cultures from each group assessed in triplicate). Data are presented as the mean \pm standard deviation.

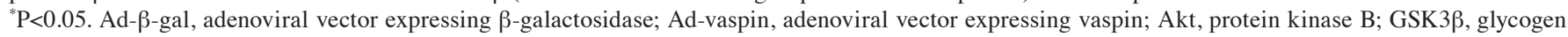
synthase kinase $3 \beta$; HPMECs, human pulmonary microvascular endothelial cells; LPS, lipopolysaccharide; rh-vaspin, recombinant human vaspin.

pathway. Taken together, these data indicated that vaspin may promote the function and refine stability of the pulmonary endothelial barrier via its anti-inflammatory, anti-apoptotic and antioxidative properties in ECs, thus protecting against LPS-induced endothelial barrier hyperpermeability and disruption in the experimental systems of ARDS. Therapeutic interventions that aim to restore vaspin levels may be favorable for the prevention or treatment of ARDS.

At present, adipose tissue is considered a significant endocrine organ that is capable of crosstalk with peripheral organs through various multifunctional adipokines (11). Compared with the well-elucidated effects of adipokines on cardiovascular disease, the association between adipokines and the pathogenesis of ARDS has only recently begun to be elucidated $(12,33)$. Given the systemic abnormalities associated with obesity and the accompanying metabolic syndrome, the effects of obesity on the pathogenesis of ARDS may be associated with numerous facets of the obese state, including dysregulation of adipokine release, which are known to contribute to the development of obesity-associated vascular disorders. The 
majority of adipokines are proinflammatory, whereas a small number of anti-inflammatory adipokines exert beneficial effects on obesity-associated complications (13). Vaspin functions as a novel predictor of obesity-associated vascular disorders and endothelial damage in human studies $(21,23)$. Furthermore, it has been demonstrated to be involved in the modulation of diverse biological process, including inflammation, apoptosis and oxidative stress in experimental studies (24-28,34), which are potentially implicated in the pathogenesis of ARDS. As the homologous gene of vaspin, $\alpha 1$-antitrypsin is well known for its function in inhibiting serum serine proteases, including neutrophil elastase, trypsin, thrombin and proteinase-3 (35). In addition, $\alpha 1$-antitrypsin has been reported to exert pleiotropic effects in the airways via its antioxidant, antiprotease, anti-inflammatory and anti-apoptotic properties (36-40). From a clinical perspective, it has previously been indicated that there is no therapeutic advantages to enhancing anti-neutrophil elastase activity in ARDS $(41,42)$. Nevertheless, to the best of our knowledge, no study has assessed the effects of vaspin on pulmonary ECs, particularly in the setting of ARDS. Therefore, the effects of vaspin on pulmonary ECs were explored in the present study.

Inflammation serves a vital role in the initiation and progression of ARDS $(43,44)$, and vaspin has been reported to exert anti-inflammatory effects against various insults $(26,34)$. The present study demonstrated that vaspin suppressed the inflammatory response to LPS in lung tissues and HPMECs, as evidenced by a decrease in the levels of proinflammatory cytokines (IL-6 and TNF- $\alpha$ ) and ICAM-1 in lung tissues, and a reduction in the activation of $N F-\kappa B$, which is a pivotal inflammatory mediator, post-LPS stimulation. Consistent with these findings, previous studies have indicated that vaspin may protect ECs from proinflammatory cytokines-induced inflammation by inhibiting activation of $\mathrm{NF}-\kappa \mathrm{B}$ and the production of its downstream molecules, including ICAM-1, VCAM-1 and MCP-1 (34). However, EC barrier hyperpermeability in ARDS involves complex interactions between ECs and numerous cell types. Furthermore, vaspin is a pleiotropic adipokine, the effects of which in obesity-associated vascular disease are not exclusive to a specific cell type via a single mechanism. Therefore, it cannot be ruled out that vaspin exerts its potential effects on other cell types, including vascular SMCs (VSMCs), polymorphonuclear leukocytes (PMN) or macrophages, all of which serve important but distinct roles in ARDS progression. Results from previous studies have indicated that vaspin has anti-inflammatory and antimigratory roles in VSMCs, and may prevent the expression of ICAM-1 in cultured VSMCs $(45,46)$. Further investigation is still required to fully elucidate the comprehensive effects of vaspin on ARDS. Taken together, although the present study did not directly detect or rule out the effects of vaspin on numerous cell types in ARDS, the present findings suggested that vaspin, as a multifunctional adipokine, exerts beneficial effects on ARDS via its anti-inflammatory function in lung endothelium.

It is widely accepted that compromised EC integrity and subsequent dysfunction of the pulmonary EC barrier contribute to pulmonary endothelial barrier hyperpermeability, thus leading to protein-rich leakage and PMN infiltration under ARDS conditions $(4,32)$. Therefore, therapeutic approaches that restore pulmonary endothelial barrier integrity and function are of vital importance in the progression of ARDS $(3,4)$. In the present study, aggravation of pulmonary microvascular hyperpermeability in a mouse model of ARDS was significantly suppressed following systemic administration of Ad-vaspin, thus suggesting that vaspin promotes endothelial barrier function in vivo, as evidenced by a significant reduction in capillary leakage (measured by BALF protein concentrations, EBDA extravasation and W/D ratios). Further in vitro investigations supported this beneficial property at the cellular level; the in vitro results demonstrated that vaspin reversed LPS-induced increases in the influx of FITC-dextran in HPMECs. The EC barrier consists of the cytoskeleton, AJs and tight junctions. Stabilization of endothelial AJs and the actin cytoskeleton is essential for a restrictive pulmonary EC barrier $(47,48)$. Notably, in the present study, no difference in AJ protein expression and cytoskeletal organization was detected in HPMECs treated with LPS. Therefore, it may be hypothesized that vaspin-induced promotion of EC barrier function is mediated by other mechanisms associated with cell integrity, rather than regulation of cell structure.

It has previously been hypothesized that a reduction in pulmonary endothelial cell apoptosis represents an important therapeutic target for ARDS (49). Vaspin has been reported to ameliorate vascular injuries under the diabetic milieu, thus exerting beneficial effects on diabetic vascular complications by promoting the proliferation and inhibiting the apoptosis of ECs (24). In addition, vaspin has been demonstrated to attenuate methylglyoxal-induced cell death in HUVECs (25). To confirm whether vaspin-induced suppression of pulmonary hyperpermeability in response to LPS is due to its protection of pulmonary ECs, the present study examined the direct effects of vaspin on pulmonary endothelium at the cellular level. Vaspin-mediated attenuation of EC barrier dysfunction was associated with a significant decrease in HPMECs apoptosis under LPS stimulation. These findings suggested that vaspin exerts its protective effects on pulmonary EC barrier during the LPS-induced ARDS milieu is dependent, at least partially, on its ability to attenuate apoptosis of ECs, thus reinforcing barrier integrity and restoring EC barrier function.

Endothelial dysfunction can be caused by various insults, among which ROS production is markedly involved in ARDS pathogenesis $(50,51)$. ROS are produced by NOX and function as second messengers of cytokines that are formed in response to various stimuli. It has previously been demonstrated that the primary downstream target of ROS is apoptosis signal-regulating kinase 1 , which regulates the balance between cell death and survival $(52,53)$. In the present study, the results demonstrated that vaspin may inhibit ROS production and NOX activation, thus alleviating damage to the pulmonary ECs barrier caused by LPS-induced oxidative burst. Consistent with these findings, vaspin has been reported to exert an antioxidative effect on various types of cells, including ECs $(25,46)$.

The present study also explored the underlying mechanism mediating the favorable effects of vaspin. The Akt-associated pathway has a central role in cell signaling with diverse cellular functions, including cell viability, proliferation, angiogenesis and migration. The Akt signaling pathway acts as an endogenous negative feedback or compensatory mechanism that serves to suppress proinflammatory and apoptotic events in response to injurious stimuli (54). According to the findings of a previous 
study, vaspin can activate Akt-associated signaling (27); therefore, the present study detected the phosphorylation levels of Akt and its substrate GSK3 $\beta$; the results confirmed that the beneficial effects mediated by vaspin in ARDS were associated with activation of the Akt/GSK3 $\beta$ signaling pathway. However, it is well known that a high degree of functional versatility and overlap exists among the Akt-associated signaling pathways, and the inflammatory response can be regulated by numerous intercellular signals that overlap with each other to modulate cell biology in an integrated manner. With regards to inflammation-associated signaling pathways, we aim to investigate those that induce activation of NF-кB, including stress-activated protein kinases/c-Jun $\mathrm{N}$-terminal kinases, p38 mitogen-activated protein kinases and extracellular signal-regulated kinases $1 / 2$ pathways, in our subsequent studies. Furthermore, although GRP78 is identified as one of the interacting molecules of vaspin on the surface of aortic ECs, further studies are required to confirm the specific mechanism that mediates the beneficial effects of vaspin on pulmonary ECs, more specifically in the pathogenesis of ARDS.

In conclusion, the present study demonstrated that vaspin protects against LPS-induced ARDS through reversing EC barrier dysfunction via the suppression of inflammation, apoptosis and ROS generation in pulmonary ECs, at least partially via activation of the Akt/GSK3 $\beta$ signaling pathway. These findings provide evidence of a causal link between vaspin and EC barrier dysfunction during ARDS, and suggest a potential therapeutic intervention for patients with ARDS in clinical practice.

\section{Acknowledgements}

The present study was supported by the National Natural Science Foundation of China (grant no. 81670071).

\section{References}

1. Ranieri VM, Rubenfeld GD, Thompson BT, Ferguson ND, Caldwell E, Fan E, Camporota L and Slutsky AS; ARDS Definition Task Force: Acute respiratory distress syndrome: The Berlin Definition. JAMA 307: 2526-2533, 2012.

2. Phua J, Badia JR, Adhikari NK, Friedrich JO, Fowler RA, Singh JM, Scales DC, Stather DR, Li A, Jones A, et al: Has mortality from acute respiratory distress syndrome decreased over time?: A systematic review. Am J Respir Crit Care Med 179: 220-227, 2009.

3. Müller-Redetzky HC, Suttorp N and Witzenrath M: Dynamics of pulmonary endothelial barrier function in acute inflammation: Mechanisms and therapeutic perspectives. Cell Tissue Res 355: 657-673, 2014

4. Lucas R, Verin AD, Black SM and Catravas JD: Regulators of endothelial and epithelial barrier integrity and function in acute lung injury. Biochem Pharmacol 77: 1763-1772, 2009.

5. Gong MN, Bajwa EK, Thompson BT and Christiani DC: Body mass index is associated with the development of acute respiratory distress syndrome. Thorax 65: 44-50, 2010.

6. Anzueto A, Frutos-Vivar F, Esteban A, Bensalami N, Marks D, Raymondos K, Apezteguía C, Arabi Y, Hurtado J, González M, et al; Ventila group: Influence of body mass index on outcome of the mechanically ventilated patients. Thorax 66: 66-73, 2011.

7. Stapleton RD and Suratt BT: Obesity and nutrition in acute respiratory distress syndrome. Clin Chest Med 35: 655-671, 2014.

8. Konter J, Baez E and Summer RS: Obesity: 'priming' the lung for injury. Pulm Pharmacol Ther 26: 427-429, 2013.

9. Matsuzawa Y, Funahashi T and Nakamura T: The concept of metabolic syndrome: Contribution of visceral fat accumulation and its molecular mechanism. J Atheroscler Thromb 18: 629-639, 2011.
10. Van Gaal LF, Mertens IL and De Block CE: Mechanisms linking obesity with cardiovascular disease. Nature 444: 875-880, 2006.

11. Yamawaki H: Mechanisms of action of novel adipocytokines on the vascular system. Nihon Yakurigaku Zasshi 137: 131-135, 2011.

12. Mattu HS and Randeva HS: Role of adipokines in cardiovascular disease. J Endocrinol 216: T17-T36, 2013.

13. Ohashi K, Shibata R, Murohara T and Ouchi N: Role of anti-inflammatory adipokines in obesity-related diseases. Trends Endocrinol Metab 25: 348-355, 2014.

14. Wang C: Obesity, inflammation, and lung injury (OILI): The good. Mediators Inflamm 2014: 978463, 2014.

15. Shah D, Romero F, Duong M, Wang N, Paudyal B, Suratt BT, Kallen CB, Sun J, Zhu Y, Walsh K, et al: Obesity-induced adipokine imbalance impairs mouse pulmonary vascular endothelial function and primes the lung for injury. Sci Rep 5: 11362, 2015.

16. Konter JM, Parker JL, Baez E, Li SZ, Ranscht B, Denzel M, Little FF, Nakamura K, Ouchi N, Fine A, et al: Adiponectin attenuates lipopolysaccharide-induced acute lung injury through suppression of endothelial cell activation. J Immunol 188: 854-863, 2012

17. Fan XF, Xue F, Zhang YQ, Xing XP, Liu H, Mao SZ, Kong XX, Gao YQ, Liu SF and Gong YS: The Apelin-APJ axis is an endogenous counterinjury mechanism in experimental acute lung injury. Chest 147: 969-978, 2015.

18. Qi D, Tang X, He J, Wang D, Zhao Y, Deng W, Deng X, Zhou G, Xia J, Zhong X, et al: Omentin protects against LPS-induced ARDS through suppressing pulmonary inflammation and promoting endothelial barrier via an Akt/eNOS-dependent mechanism. Cell Death Dis 7: e2360, 2016.

19. Hida K, Wada J, Eguchi J, Zhang H, Baba M, Seida A, Hashimoto I, Okada T, Yasuhara A, Nakatsuka A, et al: Visceral adipose tissue-derived serine protease inhibitor: A unique insulin-sensitizing adipocytokine in obesity. Proc Natl Acad Sci USA 102: 10610-10615, 2005.

20. Dimova R and Tankova T: The role of vaspin in the development of metabolic and glucose tolerance disorders and atherosclerosis. BioMed Res Int 2015: 823481, 2015.

21. Kobat MA, Celik A, Balin M, Altas Y, Baydas A, Bulut M, Aydin S, Dagli N, Yavuzkir MF and Ilhan S: The investigation of serum vaspin level in atherosclerotic coronary artery disease. J Clin Med Res 4: 110-113, 2012.

22. Aust G, Richter O, Rohm S, Kerner C, Hauss J, Klöting N, Ruschke K, Kovacs P, Youn BS and Blüher M: Vaspin serum concentrations in patients with carotid stenosis. Atherosclerosis 204: 262-266, 2009.

23. Wang HH and Wang QF: Low vaspin levels are related to endothelial dysfunction in patients with ankylosing spondylitis. Braz J Med Biol Res 49: e5231, 2016.

24. Nakatsuka A, Wada J, Iseda I, Teshigawara S, Higashio K, Murakami K, Kanzaki M, Inoue K, Terami T, Katayama A, et al: Visceral adipose tissue-derived serine proteinase inhibitor inhibits apoptosis of endothelial cells as a ligand for the cell-surface GRP78/voltage-dependent anion channel complex. Circ Res 112: 771-780, 2013.

25. Phalitakul S, Okada M, Hara Y and Yamawaki H: Vaspin prevents methylglyoxal-induced apoptosis in human vascular endothelial cells by inhibiting reactive oxygen species generation. Acta Physiol (Oxf) 209: 212-219, 2013.

26. Jung CH, Lee MJ, Kang YM, Lee YL, Yoon HK, Kang SW, Lee WJ and Park JY: Vaspin inhibits cytokine-induced nuclear factor-kappa B activation and adhesion molecule expression via AMP-activated protein kinase activation in vascular endothelial cells. Cardiovasc Diabetol 13: 41, 2014.

27. Jung CH, Lee WJ, Hwang JY, Seol SM, Kim YM, Lee YL and Park JY: Vaspin protects vascular endothelial cells against free fatty acid-induced apoptosis through a phosphatidylinositol 3-kinase/Akt pathway. Biochem Biophys Res Commun 413: 264-269, 2011.

28. Lin Y, Zhuang J, Li H, Zhu G, Zhou S, Li W, Peng W and Xu Y: Vaspin attenuates the progression of atherosclerosis by inhibiting ER stress-induced macrophage apoptosis in apoE/mice. Mol Med Rep 13: 1509-1516, 2016.

29. National Research Council (US) Institute for Laboratory Animal Research: Guidance for the description of animal research in scientific publications. Washington (DC): National Academies Press (US); 2011

30. Matute-Bello G, Downey G, Moore BB, Groshong SD, Matthay MA, Slutsky AS, Kuebler WM; Acute Lung Injury in Animals Study Group: An official American Thoracic Society workshop report: features and measurements of experimental acute lung injury in animals. Am J Respir Cell Mol Biol 44: 725-738, 2011. 
31. Livak KJ and Schmittgen TD: Analysis of relative gene expression data using real-time quantitative PCR and the 2(-Delta Delta C(T)) Method. Methods 25: 402-408, 2001.

32. Ryan D, Frohlich S and McLoughlin P: Pulmonary vascular dysfunction in ARDS. Ann Intensive Care 4: 28, 2014.

33. Shibata R, Ohashi K, Murohara T and Ouchi N: The potential of adipokines as therapeutic agents for cardiovascular disease. Cytokine Growth Factor Rev 25: 483-487, 2014.

34. Liu S, Dong Y, Wang T, Zhao S, Yang K, Chen X and Zheng C: Vaspin inhibited proinflammatory cytokine induced activation of nuclear factor-kappa B and its downstream molecules in human endothelial EA.hy926 cells. Diabetes Res Clin Pract 103: 482-488, 2014

35. Taylor NJ and Shawcross DL: Alpha1-antitrypsin deficiency. N Engl J Med 361: 2102, author reply 2102, 2009.

36. Tumen J, Meyrick B, Berry L Jr and Brigham KL: Antiproteinases protect cultured lung endothelial cells from endotoxin injury. J Appl Physiol (1985) 65: 835-843, 1988.

37. Feng Y, Hu L, Xu Q, Yuan H, Ba L, He Y and Che H: Cytoprotective role of alpha-1 antitrypsin in vascular endothelial cell under hypoxia/reoxygenation condition. J Cardiovasc Pharmacol 66: 96-107, 2015.

38. Stockley RA: The multiple facets of alpha-1-antitrypsin. Ann Transl Med 3: 130, 2015.

39. Jie Z, Cai Y, Yang W, Jin M, Zhu W and Zhu C: Protective effects of alpha 1-antitrypsin on acute lung injury in rabbits induced by endotoxin. Chin Med J (Engl) 116: 1678-1682, 2003.

40. Petrache I, Fijalkowska I, Medler TR, Skirball J, Cruz P, Zhen L, Petrache HI, Flotte TR and Tuder RM: $\alpha-1$ antitrypsin inhibits caspase-3 activity, preventing lung endothelial cell apoptosis. Am J Pathol 169: 1155-1166, 2006.

41. Wewers MD, Herzyk DJ and Gadek JE: Alveolar fluid neutrophil elastase activity in the adult respiratory distress syndrome is complexed to alpha-2-macroglobulin. J Clin Invest 82: 1260-1267, 1988.

42. Gadek JE and Pacht ER: The interdependence of lung antioxidants and antiprotease defense in ARDS. Chest 110 (Suppl 6): 273S-277S, 1996.

43. Gando S, Kameue T, Matsuda N, Sawamura A, Hayakawa M and Kato H: Systemic inflammation and disseminated intravascular coagulation in early stage of ALI and ARDS: Role of neutrophil and endothelial activation. Inflammation 28 237-244, 2004.

44. Meduri GU, Annane D, Chrousos GP, Marik PE and Sinclair SE: Activation and regulation of systemic inflammation in ARDS: Rationale for prolonged glucocorticoid therapy. Chest 136: 1631-1643, 2009.
45. Phalitakul S, Okada M, Hara Y and Yamawaki H: A novel adipocytokine, vaspin inhibits platelet-derived growth factor-BB-induced migration of vascular smooth muscle cells. Biochem Biophys Res Commun 423: 844-849, 2012.

46. Phalitakul S, Okada M, Hara Y and Yamawaki H: Vaspin prevents TNF- $\alpha$-induced intracellular adhesion molecule-1 via inhibiting reactive oxygen species-dependent NF- $\mathrm{KB}$ and PKC $\theta$ activation in cultured rat vascular smooth muscle cells. Pharmacol Res 64: 493-500, 2011.

47. Kása A, Csortos C and Verin AD: Cytoskeletal mechanisms regulating vascular endothelial barrier function in response to acute lung injury. Tissue Barriers 3: e974448, 2015.

48. Mehta D and Malik AB: Signaling mechanisms regulating endothelial permeability. Physiol Rev 86: 279-367, 2006.

49. Galani V, Tatsaki E, Bai M, Kitsoulis P, Lekka M, Nakos G and Kanavaros P: The role of apoptosis in the pathophysiology of Acute Respiratory Distress Syndrome (ARDS): An up-to-date cell-specific review. Pathol Res Pract 206: 145-150, 2010.

50. Lang JD, McArdle PJ, O'Reilly PJ and Matalon S: Oxidant-antioxidant balance in acute lung injury. Chest 122 (Suppl 6): 314S-320S, 2002

51. Tasaka S, Amaya F, Hashimoto S and Ishizaka A: Roles of oxidants and redox signaling in the pathogenesis of acute respiratory distress syndrome. Antioxid Redox Signal 10: 739-753, 2008.

52. Shiizaki S, Naguro I and Ichijo H: Activation mechanisms of ASK1 in response to various stresses and its significance in intracellular signaling. Adv Biol Regul 53: 135-144, 2013.

53. Matsuzawa A, Nishitoh H, Tobiume K, Takeda K and Ichijo H: Physiological roles of ASK1-mediated signal transduction in oxidative stress- and endoplasmic reticulum stress-induced apoptosis: advanced findings from ASK1 knockout mice. Antioxid Redox Signal 4: 415-425, 2002.

54. Manukyan MC, Weil BR, Wang Y, Abarbanell AM, Herrmann JL, Poynter JA and Meldrum DR: The phosphoinositide-3 kinase survival signaling mechanism in sepsis Shock 34: 442-449, 2010

This work is licensed under a Creative Commons Attribution-NonCommercial-NoDerivatives 4.0 International (CC BY-NC-ND 4.0) License. 